\title{
Network Representation Learning-based Drug Mechanism Discovery and Anti-inflammatory Response against COVID-19
}

\author{
Xiaoqi Wang ${ }^{1}$, Bin Xin ${ }^{1}$, Zhijian Xu' ${ }^{2}$, Kenli Li ${ }^{1,3}$, Fei Li ${ }^{4 *}$, Wu Zhong ${ }^{5 *}$, Weihong Tan ${ }^{6,7,8 *}$, \\ and Shaoliang Peng $1,3,9,10 *$
}

${ }^{1}$ College of Computer Science and Electronic Engineering, Hunan University, Changsha 410082, China.

2 CAS Key Laboratory of Receptor Research; Drug Discovery and Design Center, Shanghai Institute of Materia Medica, Chinese Academy of Sciences, Shanghai, 201203, China.

${ }^{3}$ National Supercomputing Centre in Changsha, Hunan University, Changsha 410082, China.

${ }^{4}$ Computer Network Information Center, Chinese Academy of Sciences, Beijing 100850, China.

${ }^{5}$ National Engineering Research Center for the Emergency Drug, Beijing Institute of Pharmacology and Toxicology, Beijing 100080, China.

${ }^{6}$ Molecular Science and Biomedicine Laboratory (MBL), State Key Laboratory for Chemo/Bio-Sensing and Chemometrics, College of Chemistry and Chemical Engineering, College of Biology, Aptamer Engineering Center of Hunan Province, Hunan University Changsha, Hunan 410082 , China.

7 Department of Chemistry and Department of Physiology and Functional Genomics, Center for Research at the Bio/Nano Interface, Health Cancer Center, UF Genetics Institute and McKnight Brain Institute, University of Florida, Gainesville, Florida 32611-7200, United States.

${ }^{8}$ Institute of Molecular Medicine (IMM), Renji Hospital, Shanghai Jiao Tong University School of Medicine, and College of Chemistry and Chemical Engineering, Shanghai Jiao Tong University, Shanghai, China.

${ }^{9}$ School of Computer Science, National University of Defense Technology, Changsha 410073, China.

${ }^{10}$ Peng Cheng Lab, Shenzhen 518000, China.

\begin{abstract}
Recent studies have been demonstrated that host immune imbalance is an important factors leading to acute respiratory distress syndrome (ARDS) in COVID-19 patients. Therefore, discovery of potential drugs and identification of their mechanisms of action for the prevention of immune imbalance in COVID-19 patients are urgently needed. In this study, we proposed a network representation learning-based methodology, termed Aldrug2cov, to discover drug mechanism and anti-inflammatory response for patients with COVID19. In Aldrug2cov, a deep bidirectional Transformer encoder network representation approach is developed to automatically learn lowdimensional vector of heterogeneous network. Using the representation vectors, Aldrug2cov identifies 40 potential targets and 24 high-confidence drugs that bind to tumor necrosis factor(TNF)- $\alpha$ or interleukin(IL)- 6 to prevent excessive inflammatory responses in COVID-19 patients. In particular, Aldrug2cov indicated that chloroquine and hydroxychloroquine are able to reduce fatality of COVID-19 patients, and that their mechanisms of action are likely mediated through their inhibition of inflammatory cytokines on top of their antiviral ability, consistent with the findings of clinical studies. In addition, the results in 5 pharmacological application suggested that Aldrug2cov significantly outperforms 5 other state-of-the-art network representation approaches, future demonstrating the availability of Aldrug2cov in drug development field. In summary, Aldrug2cov is practically useful for accelerating COVID-19 therapeutic development. The source code and data can be downloaded from https://github.com/pengsl-lab/Aldrug2cov.git.
\end{abstract}

Index Terms—heterogeneous networks, representation learning, deep bidirectional Transformer, COVID-19, drug mechanism, anti-inflammatory response

\section{INTRODUCTION}

G LOBALLY as of 15 June, 2020, there have been over 8,061,550 confirmed cases of COVID-19, including 440,290 deaths, reported to World Health Organization (WHO), implying that the novel coronavirus (SARS-CoV-2) has posed a global health threat(https://covid19. who.int/). In addition, it has been well proven that host immune responses are important factors leading to life-threatening acute respiratory distress syndrome (ARDS) in COVID-19

- *To whom correspondence should be addressed: Fei Li (pittacus@gmail.com), Wu Zhong (zhongwu@bmi.ac.cn), Weihong Tan (tan@chem.ufl.edu) and Shaoliang Peng (slpeng@hnu.edu.cn) patients [1]. Although numerous of researchers are devoted to elucidating the pathogenic mechanisms of SARS-CoV2 , and to developing effective medications for controlling and preventing COVID-19, Considering that the new drug development is a complex, lengthy and expensive process, one effective method of drug discovery is to apply a drug repositioning [2] strategy to identify the potential drugs among existing ones. Compared to developing a drug de novo, discovering potential drugs from existing ones may significantly reduce the cost and period of drug development. Therefore, drug repositioning has received increased attention from pharmaceutical companies, governments agencies 
and academic researchers in recent year. Nevertheless, the development of promising drug discovery approaches for the effective treatment of COVID-19 is challenging, because of insufficient knowledge regarding drug targets and the disease pathology. Therefore, it is important to understand how SARS-CoV-2 give elicits host immune responses, and to apply this knowledge towards the discovery of potential targets and drugs, and elucidation of drug mechanisms of action against COVID-19.

Identification of the proteins involved in COVID-19 is a primary step towards revealing the underlying molecular mechanisms of SARS-CoV-2 infection, and can potentially improve clinical therapies for COVID-19. Unfortunately, limited knowledge regarding the detailed pathogenic mechanism of SARS-CoV-2 has prevented comprehensive identification and evaluation of disease-causing proteins. However, a growing body of research suggests that the clinical manifestations of severe acute respiratory syndrome (SARS) and COVID-19 was highly similar, and they may have similar pathogenesis [3]. In addition, phylogenetic analysis has revealed that the genome of SARS-CoV-2 is similar to that of SARS-CoV, with approximately $79 \%$ sequence identity [4]. Above all, they have the similar hostcell receptor usage and cell entry mechanism [5]. Given these apparent similarities between the two viruses and the corresponding diseases, identification of COVID-19-related proteins based on previous SARS studies is an important step towards understanding the nature of COVID-19 and determining a possible cure for the disease.

Since the COVID-19 outbreak, many studies have focused on identifying proteins or drugs related to the entry, fusion, and replication of SARS-CoV-2. For example, scientists have demonstrated that SARS-CoV-2 uses angiotensinconverting enzyme 2 (ACE2) [6], [7] and transmembrane protease serine 2 (TMPRSS2) for entry into host cells [8]. In addition, several antiviral drugs with inhibitory effects against SARS-CoV-2 have been selectively tested in clinical trials [9], [10]. However, recent reports show that the development of severe disease does not seem to be solely related to viral load [11], and that the hyperinflammatory response induced by SARS-CoV-2 is a main cause of severe disease and death in infected patients [12]. Unfortunately, the efficacy of existing antiviral agents, such as favipiravir, arbidol, and darunavir, which are being tested in ongoing clinical studies on COVID-19, might be unsatisfactory or insufficient for patients suffering from immune imbalance, and the mechanisms of action of these drugs in this disease are uncertain [13]. Therefore, aside from the development of an antiviral treatment strategy, proteins that cause excessive inflammation, should be identified, and targeted to discover anti-inflammatory agents, particularly for the patients with severe disease.

Drug repositioning and discovery poses formidable challenges because the pharmacological action mechanisms and biological process are complex and elusive. Fortunately, with the rapid development of the systems biology and network pharmacology fields, the drug research paradigm has changed from the linear mode of "one drug, one target, one disease" to the network mode of "multi-drugs, multitargets, multi diseases" [14]. Cheng et al. have suggested that the integration of multiple perspectives network contributes to understanding and analysis of molecular action mechanisms [14]. Among the advances, network-based methods have already offered promising insights to improve the accuracy of in silico drug discovery, and to elucidate action mechanisms for the effective treatment of COVID-19 [15].

In this study, we proposed a network representation learning-based drug mechanism discovery and antiinflammatory response, termed AIdrug2cov, to identify potential drugs for COVID-19. In AIdrug2cov, a multilayer bidirectional Transformer encoder network representation approach is developed to automatically learn lowdimensional embedding vector from a heterogeneous network systematically integrating diverse drug-related information. In this study, based on the representations, AIdrug2cov identified 40 potential targets related to COVID-19, and 24 high-confidence drugs binding to TNF- $\alpha$ or IL-6 for preventing excessive inflammatory response in patients with COVID-19. Importantly, this study indicated that chloroquine (CQ) and hydroxychloroquine (HCQ) can reduce death risk of COVID-19 patients, and that the mechanism of action (MOA) of these drugs is likely mediated through its inhibition of inflammatory cytokines on top of their ability in inhibiting viral replication. This result of anti-inflammatory action of CQ and HCQ have been verified by clinical studies [16]. To evaluate and interpret the representation performance of AIdrug2cov, we integrated 3 type of pharmacological tasks: drug-drug interaction network (DDI) reconstruction, Anatomical Therapeutic Chemical (ATC) classification, and bio-link prediction. The results demonstrate that AIdrug2cov significantly outperforms 5 other state-of-the-art network representation approaches. In summary, AIdrug2cov is a practically useful tool for accelerating COVID-19 therapeutic development.

\section{Result}

\subsection{Overview of Aldrug2cov}

An overview of the proposed AIdrug2cov, which is a network representation learning-based methodology to discover drug mechanism and anti-inflammatory response for patients with COVID-19, is shown in Fig.1. First, we constructed a comprehensive heterogeneous network to integrate drugs, diseases, proteins, and side-effects. A network representation approach based on semantic paths and deep bidirectional Transformer encoder model was developed to automatically learn a low-dimensional embedding vector by systematically integrating the semantic relation and topological structure of a heterogeneous network. Then, the low-dimensional vector of nodes was fed into an inductive matrix completion (IMC) model [17] to identify the top 45 potential targets related to SARS or COVID-19. Enrichr [18] was used to perform functional enrichment analysis, and we conducted a mechanism of action (MOA) analysis based on the literature search. Note that target identification of COVID-19 is conducted with SARS data, since the clinical manifestation and pathogeneses of these diseases are highly similar [3]. Similarly, AIdrug2cov predicted 40 high-confidence drugs based on the predicted targets TNF$\alpha$ and IL-6. Next, we performed Connectivity Map (CMap) [19] analysis and literature search to identify 25 agents that bind to TNF- $\alpha$ or IL- 6 to prevent cytokine storms and 


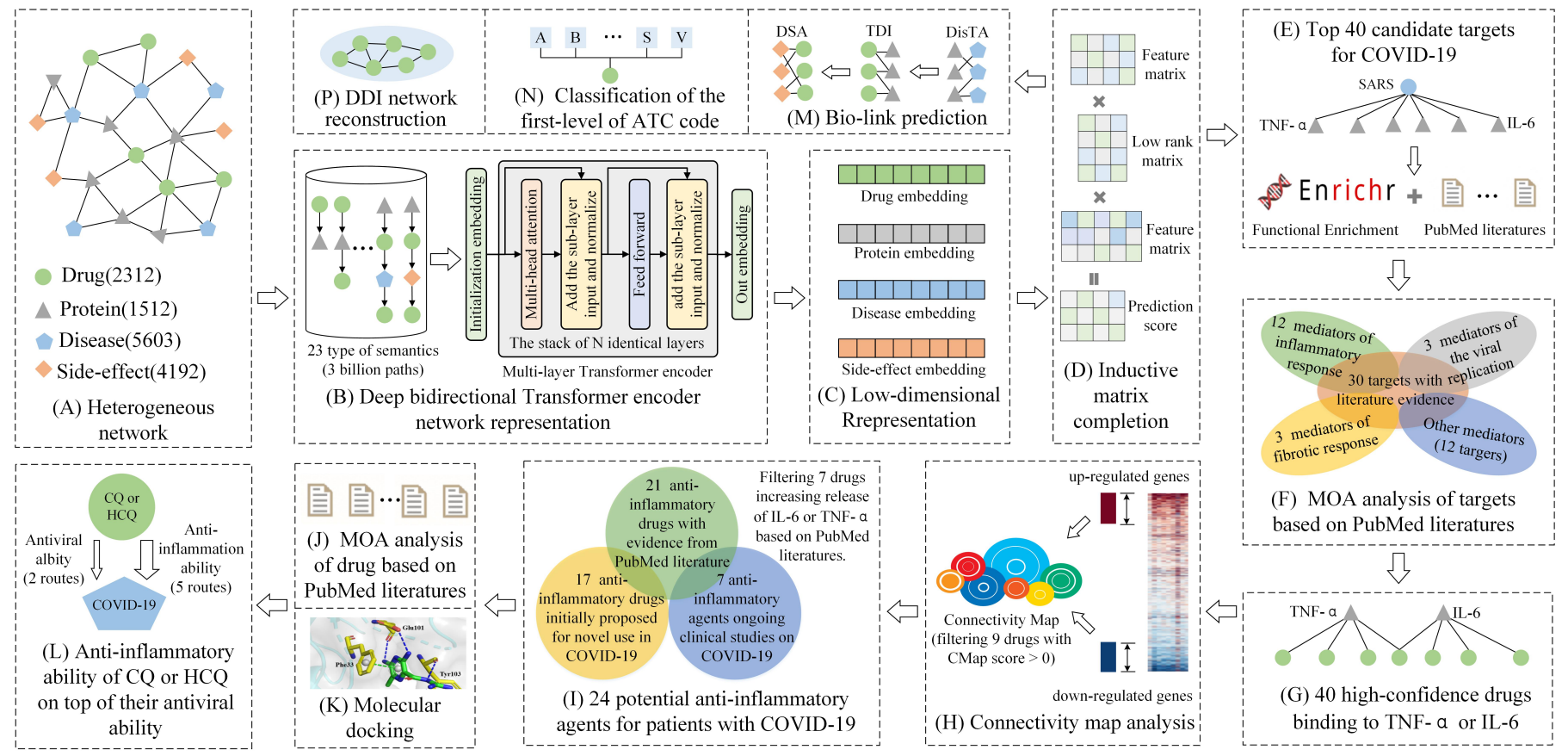

Fig. 1. Overview of Aldrug2cov to identify drug mechanism of action and anti-inflammatory response against COVID-19.

excessive inflammatory responses in patients with COVID19. Finally, we analyzed multiple mechanisms of action base on literature reports, and explored the possible binding modes between the new predicted drugs and TNF-/IL-6 with the docking program DOCK6.8 [20]. In addition, this study integrated DDI network reconstruction, ATC classification, and bio-link (i.e., disease $\rightarrow$ protein $\rightarrow$ drug $\rightarrow$ sideeffect association) prediction to future evaluate and validate the applicability, and scalability of AIdrug2cov in the drug development process.

\subsection{COVID-19 target identification}

In this study, the top 40 proteins, which corresponded to roughly $3 \%$ of the total number of the protein entity in heterogeneous network, were regarded as potential targets for COVID-19. In this study, Evidence from the literature was used to determine the action mechanism between each target and COVID-19. As shown in Table 1, most of the proteins have been demonstrated to have interaction mechanism or therapeutic associations with SARS or COVID-19. Apparently, the most popular targets seem to be mediators of inflammation, such as TNF [1], [21], IL1B [22], IL-6 [23], [24], CCL2 [22], [25], IFNG [24], [26], [27], and CXCL10 [1], [22]. The targets MAPK3 [28], TP53 [29], and RB1 [30], [31] affect the replication processes of SARS-CoV and SRAS-CoV-2. CASP3 [32], [33] and CYCS [34], [35] induce apoptosis of cells infected with SARS-CoV. AKT1 signaling pathways also play key roles in persistent SARS-CoV infection [36]. Inhibiting EGFR and TGFB1 [37], [38] signaling may prevent an excessive fibrotic response to SARS-CoV and other respiratory viral infections [39]. A GSTM1 null genotype may increase the risk of pulmonary fibrosis of COVID-19 patients [40], and it is worth noting that there has also been one report of a newly emerged fibrosis in a COVID-19 patient [41]. COVID-19 was found to aggravate already compromised NO production in a cohort with NOS3 polymorphism, and management of NOS3/iNOS ratios and NO levels can prevent the development of severe ARDS. In addition, certain crucial proteins are associated with SARSCoV or SARS-CoV-2, such as, VEGFA [42], RAC1 [38], INS [43], ICAM1 [44], [45], and CDK2 [46], [47].

\subsubsection{Inflammatory response-related targets for COVID-19}

Twelve targets appear to be critical mediators of the inflammatory response in moribund COVID-19 patients, and this response is closely related to the severity of the disease. Similar to the situation in SARS-CoV, the levels of TNF [1], [21], IL1B [22], IL-6 [23], [24], CCL2 [22], [25], IFNG [24], [26], [27] and CXCL10 [1], [22] are significantly elevated and are associated with adverse clinical outcomes in patients with COVID-19. NFKB1 is a key factor in the hyperactivation of monocyte-derived macrophages in COVID-19 [22], which directly affects the inflammatory response. The most important signal transduction pathways activated by viruses leading to the expression of proinflammatory cytokines are mediated by the factors IRF-3 and IRF-7 and JUN [48]. PARP plays a critical role in cytokine release in response to any lung injury causing viral infection, and the course of COVID-19 may be altered by inhibiting this protein [49]. HMOX1 has been shown to display anti-inflammatory properties in models of acute pulmonary inflammation, and is expressed in most cell types in organisms [50], [51]. A large body of evidence from preclinical studies indicates that MAPK14 paly a crucial role in inflammatory cytokine production [33], [52], [53]. MMP2 is a marker that aggravates pulmonary damage in SARS patients, and doxycycline markedly suppresses the levels of proinflammatory cytokines by inhibiting this protein [54], [55], [56]. PPAR is a key regulators of inflammation, and its activation results in reductions in inflammatory cytokine levels. 
TABLE 1

Candidate targets and their interaction mechanisms with COVID-19.

\begin{tabular}{|c|c|c|c|c|}
\hline NO. & UniProt ID:name & Confidence & Interaction mechanism to COVID-19 & References \\
\hline 1 & P01375:TNF- $\alpha^{*}$ & 0.5962 & Cytokine in moribund COVID-19 patients & {$[1],[21]$} \\
\hline 2 & P35354:PTGS2* & 0.5900 & A key mediator of inflammation in SARS & [57], [58], [59] \\
\hline 3 & P42574:CASP3 & 0.5811 & Critical medium inducing apoptosis of cells infected with SARS-CoV & [32], [33] \\
\hline 4 & P10415:BCL2 & 0.5667 & Necessary for survival of persistently SARS-CoV-infected cells & [60] \\
\hline 5 & P01584:IL1B* & 0.5635 & Cytokine in moribund COVID-19 patients & [22] \\
\hline 6 & P27361:MAPK3 & 0.5628 & Participant in SARS-CoV replication & [28] \\
\hline 7 & P04637:TP53 & 0.5626 & Antagonist of coronavirus replication & [29] \\
\hline 8 & P05412:JUN* & 0.5368 & Induction of proinflammatory cytokines of coronavirus & [48] \\
\hline 9 & P28482:MAPK1 & 0.5367 & NA & NA \\
\hline 10 & P99999:CYCS & 0.5337 & Medium inducing apoptosis related to SARS-CoV membrane protein & {$[34],[35]$} \\
\hline 11 & P37231:PPARG* & 0.5288 & Key regulators of inflammation & [61] \\
\hline 12 & P19838:NFKB1* & 0.5282 & Hyperactivation of monocytederived macrophages in COVID-19 & {$[22],[62]$} \\
\hline 13 & P09210:GSTA2 & 0.5204 & A factor of pulmonary fibrosis in COVID-19 patients & [40] \\
\hline 14 & P05231:IL-6* & 0.5170 & Cytokine storm in moribund COVID-19 patients & [23], [24] \\
\hline 15 & Q03181:PPARD & 0.5073 & NA & NA \\
\hline 16 & P15692:VEGFA & 0.5051 & A key factor in both ICU and non-ICU COVID-19 patients & [42] \\
\hline 17 & P09874:PARP1* & 0.5003 & An pivotal role on cytokine release in COVID-19 & [49] \\
\hline 18 & P35228:NOS2 & 0.4991 & Inhibits viral protein and RNA synthesis & {$[63],[64]$} \\
\hline 19 & P05164:MPO & 0.4945 & Higher levels of MPO in adult patients with COVID-19 & [65] \\
\hline 20 & P09601:HMOX1* & 0.4920 & Anti-inflammatory effects on LPS-induced pulmonary inflammation. & [50], [51] \\
\hline 21 & P17302:GJA1 & 0.4827 & NA & NA \\
\hline 22 & P01308:INS & 0.4824 & Obesity-related comorbidities and mechanisms of a severe course of COVID-19 & [43] \\
\hline 23 & P01137:TGFB1 & 0.4800 & Relation to the fibrosis and fluid homeostasis in the lungs for the severe COVID-19 & [37], [38] \\
\hline 24 & P00533:EGFR & 0.4771 & High rate of pulmonary fibrosis & [41], [66] \\
\hline 25 & Q16539:MAPK14* & 0.4750 & Key signaling molecules as therapeutic targets for inflammatory diseases in SARS & [33], [52], [53] \\
\hline 26 & P05067:APP & 0.4746 & NA & NA \\
\hline 27 & P45983:MAPK8 & 0.4742 & NA & NA \\
\hline 28 & P13500:CCL2* & 0.4698 & Inflammatory chemokine storms in severe COVID-19 patients & [22], [25] \\
\hline 29 & P05362:ICAM1 & 0.4689 & Key hub genes involved in COVID-19 & {$[44],[45]$} \\
\hline 30 & P63000:RAC1 & 0.4648 & A role in SARS-PLpro-induced STAT6 nuclear translocation & [38] \\
\hline 31 & P31749:AKT1 & 0.4608 & A key role in persistent SARS-CoV infection & [36] \\
\hline 32 & P06400:RB1 & 0.4607 & Initiation of gene expression and viral replication & [30], [31] \\
\hline 33 & P08253:MMP2* & 0.4601 & A marker of inflammation aggravating pulmonary damage in SARS patients & [54], [55], [56] \\
\hline 34 & P08684:CYP3A4 & 0.4579 & NA & NA \\
\hline 35 & P07101:TH & 0.4565 & NA & NA \\
\hline 36 & O75469:NR1I2 & 0.4562 & NA & NA \\
\hline 37 & P09488:GSTM1 & 0.4560 & Aggrandizement the risk of pulmonary fibrosis in COVID-19 patients & [40] \\
\hline 38 & P02778:CXCL10* & 0.4558 & Inflammatory chemokines in COVID-19 patients & [1], [22] \\
\hline 39 & P01579:IFNG* & 0.4539 & A key mediator of inflammation in COVID-19 patients & [24], [26], [27] \\
\hline 40 & P24941:CDK2 & 0.4538 & N-protein of SARS-CoV inhibition of CDK2 activity & [46], [47] \\
\hline
\end{tabular}

Proteins marked with $*$ may be key mediators of inflammation in COVID-19.

\subsubsection{KEGG and GO enrichment analyses of targets}

We use Enrichr [18] tool to perform Kyoto Encyclopedia of Genes and Genomes (KEGG) and Gene Ontology (GO) enrichment analyses to comprehensively evaluate the biological relevance and functional pathways of COVID-19 targets predicted by AIdrug2cov. KEGG pathway enrichment analysis revealed the top 10 significant biological pathways (ranked with $p$-values according to the guidelines in [18]), which included TNF signaling pathway, epstein-Barr virus infection, the IL-17 signaling pathway, and the human cytomegalovirus infection pathway, as shown in Fig.2(A). Similarly, GO biological process enrichment analysis further confirmed that the targets were associated with multiple processes related to host cell lifecycle and viral replication, including cytokine activity, MAP kinase activity, transcription regulatory region DNA binding, protein kinase activ- ity, RNA polymerase II (RNAPII) regulatory region DNA binding, RNAPII transcription factor activity, and sequencespecific transcription regulatory region DNA binding as shown in Fig.2(B).

Based on the above results, we concluded that most of the candidate proteins predicted by AIdrug2cov were targeted by SARS-CoV-2 and targetable for COVID-19 treatment. This motivated us to develop a drug repurposing strategy by specifically targeting host proteins for potential treatment of COVID-19.

\subsection{Hyperinflammation in patients with COVID-19}

Indeed, several recent COVID-19 clinical studies have shown that SARS-CoV-2 induces excessive and aberrant host immune responses that are associated with severe lung 


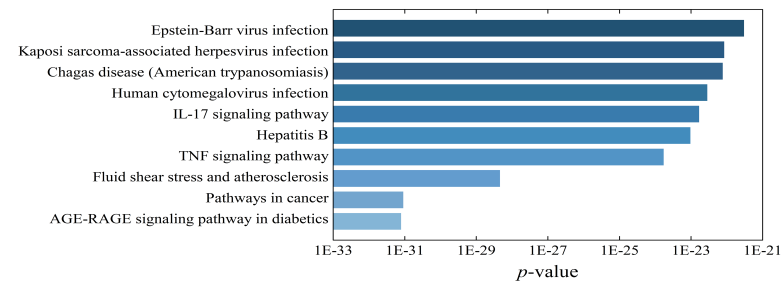

(A) KEGG human pathway analyses

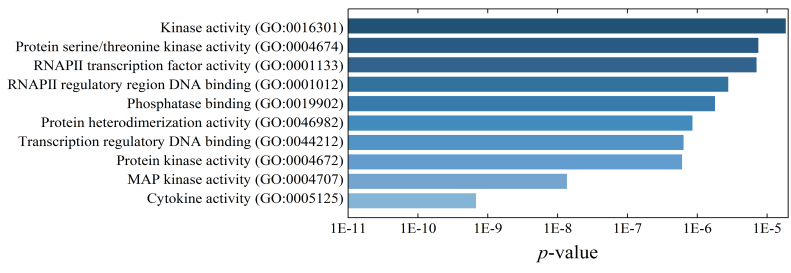

(B) GO enrichment analyses

Fig. 2. KEGG human pathway and GO enrichment analyses for the potential COVID-19 target proteins

pathology, leading to death [1]. Based on previous literaturereported knowledge, we find that the production of cytokines, such as IL- 6 , and TNF- $\alpha$, is increased in patients with severe COVID-19. This is similar to the case in patients with SARS-CoV, indicating that SARS-CoV-2 infection is associated with a cytokine storm and severe pulmonary inflammation in moribund patients.

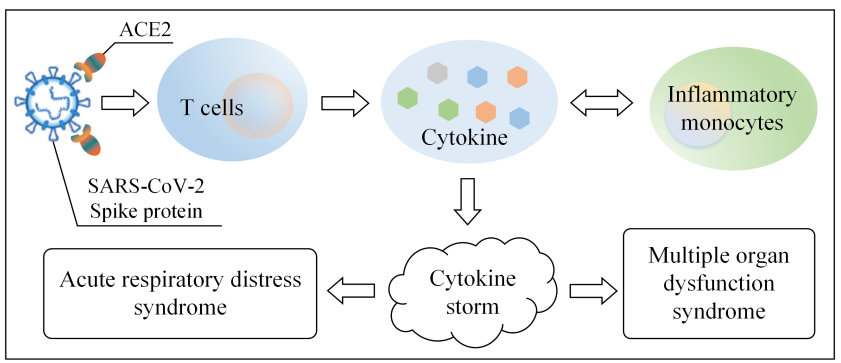

Fig. 3. Possible pathway contributing to excessive inflammatory responses in patientS with COVID-19.

A possible pathway contributing to excessive host inflammatory responses in COVID-19 is shown in Fig.3. First, SARS-CoV-2 is taken up into a host-cell by binding ACE2. Then, $\mathrm{T}$ lymphocytes are excessively activated and generate large amounts cytokines such as TNF- $\alpha$, IL-6, and GMCSF. The cytokine-rich environment induces inflammatory monocytes with high express of cytokine, and further accelerates the inflammatory response. The aberrantly activated $\mathrm{T}$ cells and inflammatory monocytes may enter the pulmonary circulation leading to a cytokine storm, thus causing ARDS or multiple organ dysfunction syndrome.

In addition, recent studies have suggested that drugs targeting IL- 6 and TNF- $\alpha$ are effective in blocking inflammatory storms, and are promising treatment agents for severe COVID-19 patients [21], [23]. Therefore, this work focused on drug discovery based on IL-6 and TNF- $\alpha$ to identify potential anti-inflammatory agents with efficacy against COVID-19.

\subsection{Anti-inflammatory drug discovery and mechanism of action analysis}

In the drug discovery step for the COVID-19, 1\% the all drugs in the heterogeneous network were selected as candidate agents binding to TNF- $\alpha$ or IL-6, respectively. Then, several drugs were filtered via CMap analysis model. We identified 31 drugs that interact with TNF- $\alpha$ or IL-6 (17 drugs that bind to TNF- $\alpha, 14$ drugs that bind to IL-6). Finally, we use subject matter expertise based on literaturereported knowledge to filter out 6 drugs, including arsenic trioxide, acetaminophen, isoflurane, halothane, latanoprost, and hydrochlorothiazide, since these drugs tend to increase release of IL- 6 or TNF- $\alpha$. For example, acetaminophen significantly increase the hepatic levels of IL-6, TNF- $\alpha$, IL-10 and monocyte chemoattractant protein [67], [68]. Isoflurane induced marked upregulation of the proinflammatory cytokines TNF- $\alpha$, IL1B, IL- 6 and IL-8 in hippocampus tissue [69]. The expression levels of IL-6, and TNF- $\alpha$ tend to increase in the birds chronically treated with arsenic trioxide [70]. Halothane potentiates alcohol adduct-induced TNF- $\alpha$ release in heart endothelial cells [71]. Latanoprost stimulated the release of IL-6 from human tendon capsule fibroblasts in a concentration-dependent and time-dependent manner [72]. Hydrochlorothiazide has not had in in vitro antiinflammatory effects at clinical studies. Meanwhile, there was a trend to increase the production of IL1B at the lower concentrations of hydrochlorothiazide [73].

Based on the above procedure, we identified 25 highconfidence drugs (13 drugs binding to TNF- $\alpha, 13$ drugs binding to IL-6) with efficacy against COVID-19 as shown in Table 2; acarbose is treat as agent that binds to both TNF- $\alpha$ and IL-6. We found that 21 drugs that have been previously reported in the literature could reduce the expression and release of TNF- $\alpha$ or IL- 6 to exert anti-inflammatory effects in silico. Although, most of these drugs are treated as potential therapeutic agents for cytokine storm inhibition, this study suggests their role in inflammatory response prevention in patients with COVID-19 for the first time.

\subsubsection{Seventeen anti-inflammatory drugs initially proposed for novel use in COVID-19 patients}

To the best of our knowledge, 17 of the drugs predicted by AIdrug2cov were initially proposed as potential therapeutic for COVID-19. The literature evidence suggests that these drugs inhibit cytokine release and the inflammatory response, as listed in column 4 in Table 2. For example, dasatinib, a small molecule Src/Abl tyrosine kinase inhibitor approved for the treatment of chronic myelogenous leukemia, reduces TNF- $\alpha$ and IL- 6 secretion in response to TLR stimulation of bone marrow-derived macrophages in vitro to modulate the host immune response [86]. Minocycline, a second generation tetracycline antibiotic, exerts its antiinflammatory effect on microglia by inhibiting the expression and release of TNF- $\alpha$, and IL1B [88]. Tranexamic acid plays a key role in reducing subsequent systemic inflammatory responses in a manner associated with reductions in the circulating levels of the proinflammatory cytokines IL-6 and IL-8 [99]. IL-6 in lung tissues in methazolamide-treated mice were markedly decreased. Methazolamide treatment has been found to markedly decrease IL-6 levels in mouse lung 
TABLE 2

Candidate drugs and their interaction mechanisms with COVID-19.

\begin{tabular}{|c|c|c|c|c|}
\hline Target ID: name & Drugbank ID: name & Confidence & Mechanism of action to COVID-19 & References \\
\hline \multirow{13}{*}{ P01375:TNF } & DB01041:Thalidomide* & 0.8719 & Decreasing stability of mRNA & [74], [75], [76], [77] \\
\hline & DB00608:Chloroquine* & 0.8336 & Playing multiple roles (see details in Section 2.5) & [78], [79], [80] \\
\hline & DB01427:Amrinone & 0.7627 & Concentration dependent manner & [81] \\
\hline & DB01611:Hydroxychloroquine* & 0.7069 & Playing multiple roles (see details in Section 2.5) & [82], [83], [84], [85] \\
\hline & DB01254:Dasatinib & 0.7034 & Unclear & [86] \\
\hline & DB00284:Acarbose & 0.6698 & Decreasing the expression of miRNAs & [87] \\
\hline & DB01017:Minocycline & 0.6458 & Unclear & [88] \\
\hline & DB00619:Imatinib & 0.6444 & Reducing DNA binding of NF- $\kappa$ B & [89] \\
\hline & DB00228:Enflurane & 0.6282 & Unclear & NA \\
\hline & DB00975:Dipyridamole* & 0.6255 & Unclear & [90], [91] \\
\hline & DB01115:Nifedipine & 0.6162 & Unclear & [92], [93] \\
\hline & DB00724:Imiquimod & 0.6145 & Unclear & [94] \\
\hline & DB00768:Olopatadine & 0.6116 & Unclear & [95], [96] \\
\hline \multirow{13}{*}{ P05231:IL-6 } & DB00198:Oseltamivir* & 0.3241 & Reducing the mRNA levels & [97], [98] \\
\hline & DB00284:Acarbose & 0.3030 & Reducing the mRNA levels & [87] \\
\hline & DB00302:Tranexamic acid & 0.3004 & Concentration dependent manner & [99] \\
\hline & DB01258:Aliskiren & 0.2984 & Reducing the mRNA levels & [100], [101] \\
\hline & DB00819:Acetazolamide & 0.2979 & Reducing the mRNA levels & [102] \\
\hline & DB00869:Dorzolamide & 0.2976 & Unclear & NA \\
\hline & DB00851:Dacarbazine & 0.2917 & Unclear & NA \\
\hline & DB00703:Methazolamide & 0.2884 & Unclear & [103] \\
\hline & DB00207:Azithromycin* & 0.2850 & Unclear & [104], [105], [106] \\
\hline & DB06228:Rivaroxaban & 0.2745 & Reducing the mRNA levels & [107], [108], [109] \\
\hline & DB00811:Ribavirin* & 0.2741 & Reducing the mRNA levels & [110], [111], [112], [113] \\
\hline & DB00594:Amiloride & 0.2676 & Unclear & [114] \\
\hline & DB01143:Amifostine & 0.2633 & Inducing activation of redox sensitive signaling & [115] \\
\hline
\end{tabular}

a. Drugs marked with $*$ have been used in clinical trials. The others are here proposed for the first time as anti-inflammatory agents for COVID-19 treatment.

b. NA represents that there have been no studies proving that the drug can inhibit the release of TNF or IL-6.

tissues, and lung inflammatory parameters and pathological changes are attenuated in methazolamide-treated mice compared with control mice [103]. Amiloride inhibits IL-6 release, and is treated as a therapeutic agent in respiratory syncytial virus infections [114]. Thus, these results from literature suggest that the proposed AIdrug2cov is able to predict drug candidates that ameliorate the cytokine storm and inflammatory response in patients with COVID-19.

\subsubsection{Anti-inflammatory effects of 7 agents in current ongo- ing clinical studies on COVID-19}

In this study, 7 of the predicted drugs have been determined in clinical studies to have antiviral activity against SARS-CoV-2, that is, thalidomide, chloroquine, hydroxychloroquine, dipyridamole, oseltamivir, azithromycin, and ribavirin. Unfortunately, only thalidomide has been used as an immunomodulator to treat patients with COVID-19 infection. Most of current studies have used other drugs to block fusion or replication of SARS-CoV-2. For example, Liu et al. found that dipyridamole suppresses SARS-CoV-2 replication at a half-maximal effective concentration $\left(\mathrm{EC}_{50}\right)$ of $100 \mu M$ in vitro [90]. Touret et al. found that azithromycine with $\mathrm{EC}_{50}>2 \mu M$ and $\mathrm{EC}_{50}<12 \mu M$ inhibits SARS-CoV-2 replication in vitro [104]. However, these drugs play an important role in anti-inflammatory effect at clinical. For example, dipyridamole performs the anti-inflammatory roles by reducing the levels of TNF in a rat model of arthritis, and prophylactic treatment reduces the arthritis-associated pathology [91]. Azithromycin significantly inhibits IL-6 and IL- 8 secretion by the TNF- $\alpha$-stimulated cystic fibrosis epithelial bronchial epithelial cells to decrease the inflammatory response [105], [106]. The levels of IL-6 and TNF- $\alpha$ are reduced in mice treated with the combination of oseltamivir and favipiravir [97]. In infected young adults, oral oseltamivir reduces nasal levels of proinflammatory cytokine, including IL-6 and TNF- $\alpha$ [98]. Apparently, these results indicate that these broad spectrum antiviral agents play important roles in the anti-inflammatory process. Therefore, ongoing clinical studies on COVID-19 should investigate the anti-inflammatory effects of these 7 agents.

\subsubsection{Anti-inflammatory actions through multiple pathways}

Among these, literature search revealed that 21 drugs are able to inhibit TNF- $\alpha$, and IL- 6 release, and reduce inflammatory responses. Strikingly, 6 drugs, including thalidomide [76], [77], chloroquine [78], [79], aliskiren [100], [101], acetazolamide [102], rivaroxaban [107], [108], [109], and ribavirin [110], exert their inhibitory action on TNF- $\alpha$, and IL-6 by decreasing mRNA stability or enhancing mRNA degradation. In addition, we found that administration of acarbose to diabetic rats significantly reduces the expression of miRNAs to inhibit the release of TNF- $\alpha$, and IL- 6 in inflammatory pathways [87]. Furthermore, amrinone reduces the release of TNF- $\alpha$ in a concentration dependent manner [81]. Imatinib inhibits TNF- $\alpha$ release by reducing the DNA binding of NFKB [89]. Amifostine is considered a thera- 
peutic agent of lung inflammation that acts by suppressing IL-6 induced activation of redox sensitive signaling [115]. Ribavirin inhibits the expression of TNF- $\alpha$, IL-6, and IL-10 in blood lymphocytes by reduced their mRNA levels [110], [111]. Notably, a study by Wang et al. has suggested that peaks in the levels of inflammatory cytokines (IL-6 and IL8) levels coincide with or occur after the peaks in SARS-CoV loads, and indicated that viral replication leads to the activation of proinflammatory cytokines, contributing to disease progression [112], [113]. These clinical findings imply that ribavirin is able to reduce the release of IL- 6 and IL- 8 by inhibiting viral replication to ameliorate lung lesions. The above studies illustrated that these drugs can ameliorate the TNF- $\alpha$ and IL-6 release to reduced inflammatory responses via multiple pathways.

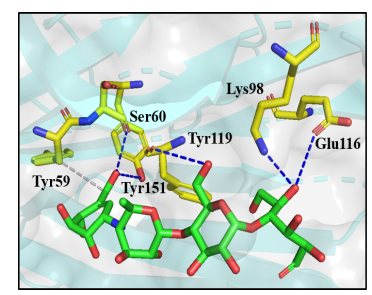

(A) Acarbose-TNF- $\alpha$

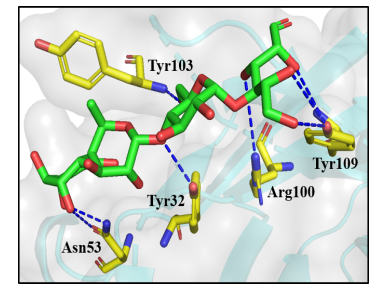

(C) Acarbose-IL-6

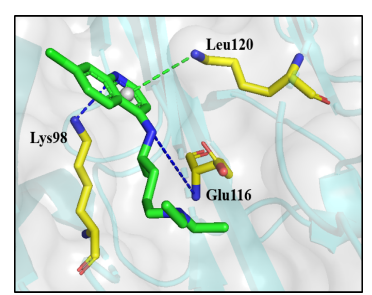

(B) Chloroquine-TNF- $\alpha$

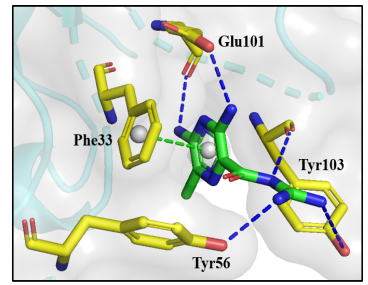

(D) Amiloride-IL-6
Fig. 4. Molecular docking results for drugs binding to TNF- $\alpha$ or IL-6. The blue, green, and gray dotted line represent hydrogen bond, $\pi-\pi$ stacking, and hydrophobic interaction between drugs and targets, respectively.

\subsubsection{Molecular docking analysis}

In this section, we used the molecular docking program DOCK6.8 [20] to explore the possible modes of binding of new the predicted drugs with TNF- $\alpha$ or IL-6. The threedimensional (3D) structures of TNF- $\alpha$ and IL-6 used in the docking studies were downloaded from the Research Collaboratory for Structural Bioinformatics (RCSB) Protein Data Bank (PDB IDs 2AZ5 and 4CNI, respectively). The $3 \mathrm{D}$ structures of the drugs were obtained from the ZINC database. Here, four representative docking structures are shown in Fig. 4

For the docking model of TNF- $\alpha$, the molecular docking result in Fig.4(A) shows that acarbose mainly binds to Ser60, Lys98, Glu116, Tyr119, and Tyr151 through five hydrogen bonds, and there is one hydrophobic interaction between acarbose and Tyr59. Fig.4(B) shows that CQ binds to TNF- $\alpha$ via two hydrogen bonds with Leu120 and Lys98, and one hydrophobic interaction with Leu120. The results indicate that there are some differences in the binding modes of the different drugs and that acarbose has a stronger ability to mainly bind TNF- $\alpha$ than CQ in terms of the hydrogen bond formation. In the docking model for IL-6, seven hydrogen bonds were predicted to form between acarbose and Tyr32,
Asn53, Arg100, Tyr103, and Tyr109 of IL-6. The result in Fig.4(D) show that Tyr56, Phe33, Glu101, and Tyr103 of IL-6 respectively combined with amiloride through five hydrogen bonds, and formed an $\pi-\pi$ stacking. The results suggest that acarbose has a stronger ability to bind IL-6 than amiloride in terms of the hydrogen bond formation. In addition, analysis of the key binding sites between acarbose and TNF- $\alpha /$ IL- 6 revealed that acarbose binds strongly and stably with these molecules through different binding sites. Based on the above results, we conclude that there are the some differences in the binding modes for the different drugs and targets.

\subsection{Mechanisms of anti-inflammatory effects of chloro- quine and hydroxychloroquine in COVID-19 patients}

In this study, chloroquine (CQ) and hydroxychloroquine (HCQ), which had high confidence scores, were regarded as agents targeting TNF- $\alpha$ and IL-6. In particularly, CQ has been added to the list of trial drugs in the Guidelines for the Diagnosis and Treatment of COVID-19 (seventh edition) published by the National Health Commission of the People's Republic of China (http://www.nhc.gov.cn/yzygj/s7653p/202003/ 46c9294a7dfe4cef80dc7f5912eb1989.shtml). CQ and HCQ are FDA-approved drugs for malaria treatment and are viral mRNA and protein synthesis inhibitors, respectively. Here, we summarize the potential anti-inflammatory mechanisms of action of CQ and HCQ based on literaturereported knowledge to accelerate therapeutic development for COVID-19.

CQ and HCQ share similar chemical structures and mechanisms of action, and demonstrate strong immunomodulatory capacity, preventing inflammation and organ damage as shown in Fig.5. Several in vitro studies have shown that CQ inhibits the production of TNF- $\alpha$ and IL- 6 via different mechanisms in human monocytes/macrophages. CQ inhibits the release of IL-6 by decreasing the stability of IL-6 mRNA [78]. In contrast, CQ has been shown to inhibit TNF- $\alpha$ synthesis mainly by blocking conversion of cellassociated $26-\mathrm{kDa}$ TNF- $\alpha$ precursor into the soluble $17-\mathrm{kDa}$ mature form, rather than by reducing the stability of TNF- $\alpha$ mRNA [78], [79], [80]. Meanwhile, one anti-inflammatory mechanism of CQ might involve impairment of antigen presentation [116]. CQ increases the intracellular $\mathrm{pH}$ and inhibits lysosomal activity in antigen-presenting cells (APCs) including monocytes, macrophages and B cells, thus preventing antigen processing and major histocompatibility complex (MHC) class II-mediated autoantigen presentation to $\mathrm{T}$ cells [117]. This process reduces $\mathrm{T}$ cell activation, thus reducing the production of cytokines including IL-6 and TNF- $\alpha$ [118]. HCQ is able to decrease the release of cytokines including IL-6 and TNF, via blocking proliferative responses to $\mathrm{T}$-cell mitogens [82], [83]. In addition to a role in immune modulation, HCQ and CQ inhibit receptor binding and membrane fusion, which are required for cell entry by coronaviruses. These drugs can interfere with the glycosylation of ACE2 of SARS-CoV to impede the binding of the virus to receptors on cells [84]. CQ increases the endosomal $\mathrm{pH}$ and inhibits protease activity such that the virus/cell fusion process is blocked [85]. 


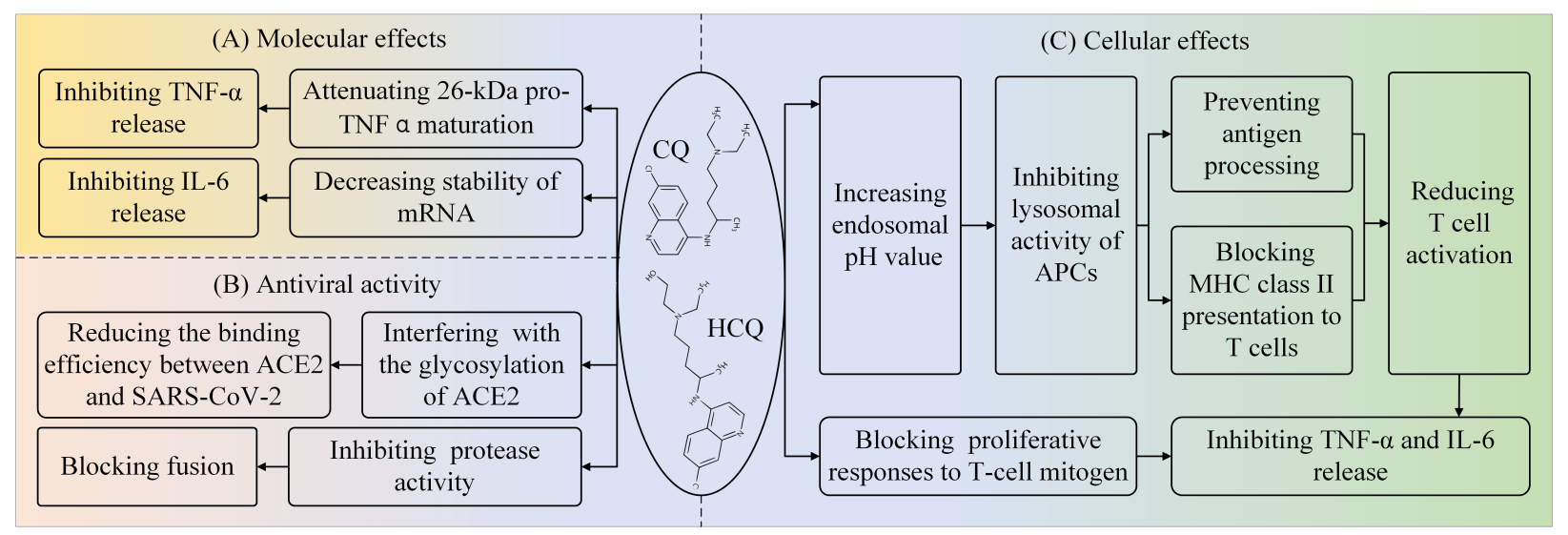

Fig. 5. Possible pathway of $C Q$ and $H C Q$ contributing to excessive inflammatory responses and antiviral effect in patients with COVID-19.

Based on the above findings, CQ and HCQ, which were identified as candidates by AIdrug2cov, can inhibit expression and release of cytokines including TNF- $\alpha$ and IL-6 via multiple mechanisms of action to block the inflammatory response. Compared to exhibiting antiviral ability, HCQ and CQ antagonize the inflammatory response through more pathways, indicating that mechanisms of action of these drugs are likely mediated through their inhibition of inflammatory cytokines on top of their antiviral ability for COVID-19 patients.

\subsection{Performance evaluation of Aldrug2cov for pharma- cological applications}

To evaluate the network representation performance of AIdrug2cov, the pharmacological interpretation results were based on comprehensively compared with those obtained from LINE [119], GraRep [120], struc2vec [121], and NeoDTI [122] models. The first three of these models have shown remarkable performance in the link prediction and node classification for 7 biomedical network datasets [123]. NeoDTI is specially designed to predict drug-target interactions, which also integrate IMC model.

- LINE: This model captures local and global network structures by approximating the 1st-order proximity and 2nd order proximity of nodes.

- GraRep: This model considers high-order proximity to preserve the network structure, and employs different loss functions to capture local relational information from the different k-step.

- struc2vec: This model uses a hierarchy to measure node similarity at different scales, and constructs a multilayer network to encode structural similarities. Then, deepwalk [124] is performed on the multilayer network to learn the low-dimensional vector of each node.

- NeoDTI: This model integrates neighborhood information of a heterogeneous network constructed from diverse data sources via a number of information passing and aggregation operations.

\subsubsection{Experiment settings and evaluation metrics}

The AIdrug2cov model parameters followed those BERT [125] which is $L=12, H=768$, and $A=12$, where $L, H$, and

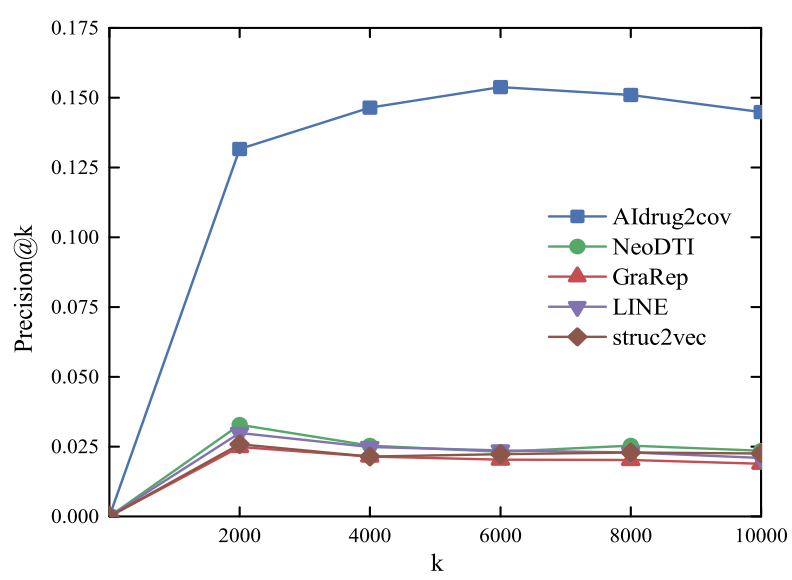

Fig.6. Precision@k from DDI network reconstruction.

$A$ are the number of Transformer blocks, the hidden size, and the number of self-attention heads, respectively. The hyperparameters of the LINE, GraRep, and struc2vec models were selected according to the guidelines in [123], because Yue et al. carefully optimized them by grid search. The hyperparameter for the NeoDTI model was set to the default value in [122]. In this study, the embedding dimension $(d)$ was set to 768 for all model.

To evaluate the performance of the embedding methods on DDI network reconstruction, we adopted Precision@k [126] as the evaluation metric. The one error, coverage, ranking loss, and average precision were used to evaluate the overall performance of all representation methods in ATC classification. These metrics are defined in detail in [127], and are frequently used for evaluating the performance of ATC classifiers. The area under precision recall (AUPR) curve and the area under receiver operating characteristic(AUROC) curve were employed to evaluate the performance of all representation methods in bio-link prediction.

\subsubsection{DDI network reconstruction}

For DDI network reconstruction, the Precision@k was calculated for different $\mathrm{k}$ values of 2,000, 4,000, 6,000, 8,000, and 10,000 , which corresponded to roughly $20 \%, 40 \%, 60 \%$, $80 \%, 100 \%$ of the total number of the DDI edges $(10,036)$, respectively. Fig. 6 illustrates the Precision@k values for the 
different $\mathrm{k}$ values. AIdrug2cov significantly outperformed the baseline methods. In addition, AIdrug2cov showed the best precision while baseline methods, when the was $k=6,000$, while the baseline methods exhibited the best reconstruction precision when $\mathrm{k}$ was 2,000. This finding indicates indicates that AIdrug2cov may reconstruct more edges than the baseline methods. This may be attributable to the capability of AIdrug2cov to learn semantic features among nodes through a deep bidirectional Transformer encoder.

\subsubsection{ATC classification}

For ATC classification, we performed 10-fold crossvalidation, in which a subset of $10 \%$ of the drug entities were randomly selected as the test set, and the remaining $90 \%$ of drugs were treated as the training set. To reduce the data bias of cross-validation, it was repeated 10 times and the average performance was computed.

Table 3 shows the results of ATC classification generated by the AIdrug2cov and baseline methods, and the best results are marked in boldface. The results clearly demonstrated that AIdrug2cov was able to achieve better results for ATC classification than the baseline methods. In particular, AIdrug2cov achieved an approximately 50\% improvement in terms of one error value. A major reason for this superiority is that AIdrug2cov takes into consideration the various types of semantic information that indicate the anatomical therapeutic chemical of drugs to a certain extent. This result indicates that AIdrug2cov is a powerful network representation method for predicting the ATC classification of given drugs.

TABLE 3

Results of ATC classification generated by Aldrug2cov and baseline methods.

\begin{tabular}{ccccc}
\hline Method & One error & Coverage & Ra-Loss & Avg-pre \\
\hline AIdrug2cov & $\mathbf{0 . 4 2 0 9}$ & $\mathbf{2 . 6 5 8 3}$ & $\mathbf{0 . 1 6 4 2}$ & $\mathbf{0 . 6 7 9 5}$ \\
NeoTDI & 0.7797 & 4.2910 & 0.2904 & 0.4211 \\
GraRep & 0.7628 & 4.2976 & 0.2905 & 0.4237 \\
LINE & 0.7685 & 4.2335 & 0.2845 & 0.4307 \\
struc2vec & 0.7981 & 4.3794 & 0.2966 & 0.4050 \\
\hline
\end{tabular}

a. Ra-Loss and Avg-pre stand for ranking-loss and average precision, respectively.

b. Among the mentioned evaluation metrics, smaller values show better performance except in the case of average precision.

\subsubsection{Bio-link prediction}

For bio-link prediction, we performed a 10 -fold crossvalidation test on all positive pairs and a matching number of randomly sampled negative pairs. Similar to prediction of ATC classification, the ratio between the test and training set was 1:9, and each method was repeated 10 times and the average performance was computed. Table 4 summarizes the overall performance of different methods for the biolink prediction, that is, disease-target association (DisTA), target-drug interaction (TDI), and drug-side-effect association (DSA).

In this DisTA, and DSA prediction tasks, AIdrug2cov outperformed the baseline methods. In particular, AIdrug2cov was significantly superior to struc2vec, improving the AUROC and AUPR by over 10\%. For
TABLE 4

Results of bio-link prediction generated by Aldrug2cov and baseline methods.

\begin{tabular}{|c|c|c|c|c|c|c|}
\hline \multirow{2}{*}{ Method } & \multicolumn{2}{|c|}{ DisTA } & \multicolumn{2}{|c|}{ DTI } & \multicolumn{2}{|c|}{ DSA } \\
\hline & AUROC & AUPR & AUROC & AUPR & AUROC & AUPR \\
\hline AIdrug2cov & 0.9613 & 0.9555 & 0.9973 & 0.9968 & 0.9391 & 0.9361 \\
\hline NeoTDI & 0.9209 & 0.9044 & 0.8782 & 0.8806 & 0.9271 & 0.9183 \\
\hline GraRep & 0.9179 & 0.8976 & 0.8050 & 0.7975 & 0.8902 & 0.8786 \\
\hline LINE & 0.9020 & 0.8846 & 0.8403 & 0.8352 & 0.8788 & 0.8684 \\
\hline struc2vec & 0.8336 & 0.7942 & 0.7514 & 0.7426 & 0.8384 & 0.8279 \\
\hline
\end{tabular}

DTI prediction, the baseline methods achieved poor results below 0.9 in terms of AUROC and AUPR, while the AIdrug2cov method showed the excellent performance with results close to 1 . These findings suggest that AIdrug2cov can still obtain good results when other methods fail to accurately predict the DTI. In addition, we observe that AIdrug2cov greatly outperform other baseline methods, with significant improvement (13\% in terms of AUPR and AUROC) over the second best method.

Interestingly, GraRep and LINE had improved the link prediction performance compared with struc2vec, whereas their result is lower than NeoDTI of ones. For example, compared with struc2vec, LINE achieved $4.8-11.2 \%$ improvement in terms of AUROC value on the 3 bio-link prediction tasks. NeoTDI achieved 5.2\% increment with regard to average AUPR in the 3 bio-link prediction tasks, when compared with GraRep. This may be because NeoDTI use a neighborhood information-preserving learning procedure to enforce the extracted feature representations of nodes to match the observed networks. There may be a lack of structural identity in the heterogeneous network, thus leading to the poor performance of struc2vec.

The proposed AIdrug2cov method clearly achieved very promising results in various prediction tasks. Three key factors were responsible. First, AIdrug2cov uses 23 types of meta-paths to integrate the structure and semantic feature among diverse vertices in the heterogeneous network. Second, although AIdrug2cov considers only the first-order proximity of nodes in the construction process of semantic paths, it can capture long-range dependencies without regard to their distance in the input or output sequences by relying entirely on an attention mechanism. Moreover, AIdrug2cov uses masked language models to enable train deep bidirectional representation.

\subsubsection{Effect of dimension}

The embedding dimension $(d)$ is a common hyperparameter among AIdrug2cov, LINE, GraRep, struc2vec, and NeoDTI. In this study, each method was run 8 times with a different embedding dimensions (i.e. $d=48,96,192,384,768,1,536$, 2,304 , and 3,072 ) to evaluate the impact of dimensionality on the prediction performance and time efficiency. Fig.7 illustrates the effects of dimension on DDI network reconstruction $(\mathrm{k}=10,000)$, ATC classification, and bio-link prediction. Generally, the prediction performance improved with increasing embedding dimensionality. The same conclusion is described in [123]. This is intuitive since higher number of dimensions can encode more useful information. 

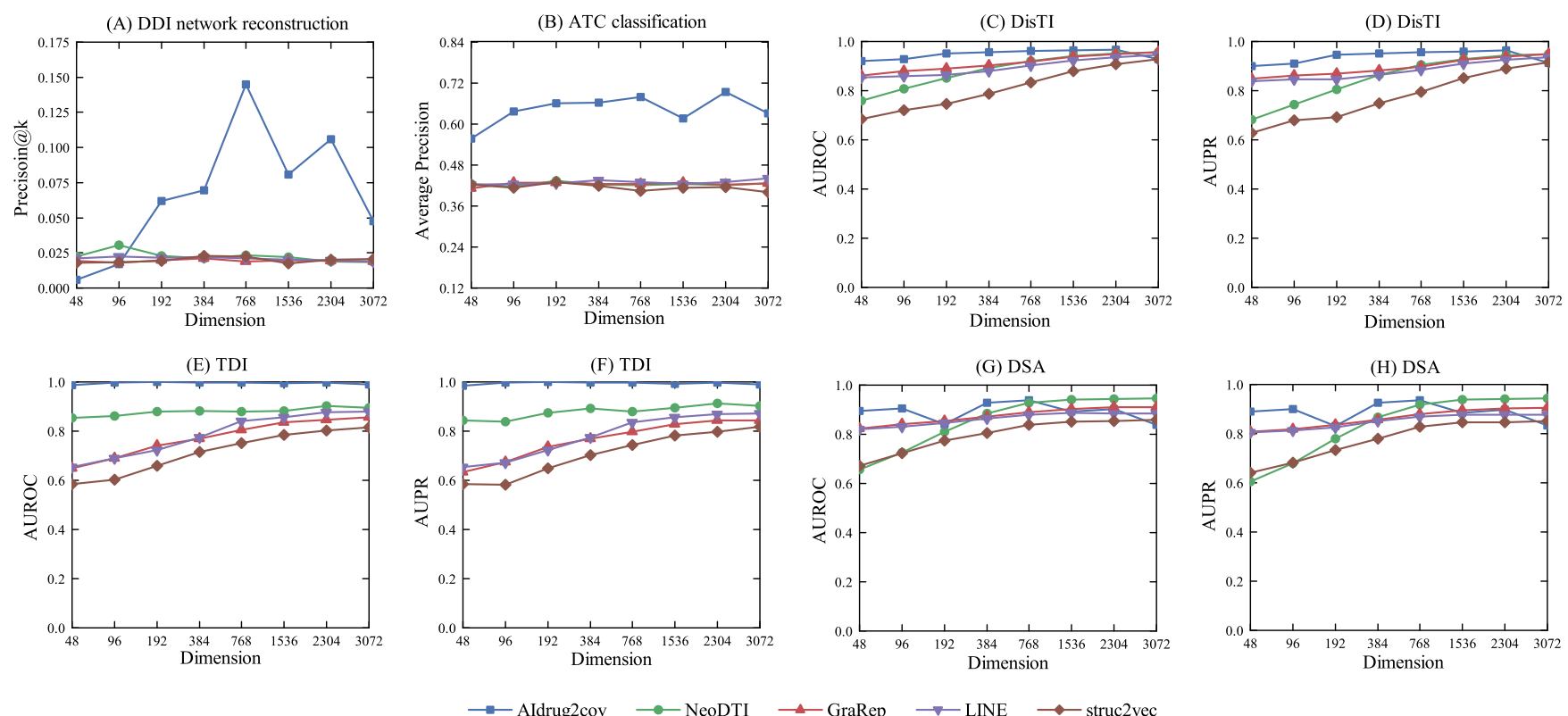

Fig. 7. Results of DDI network reconstruction; ATC classification; DisTI, TDI, and DSel with different embedding dimensions.

However, the performance tends to saturate or decrement when the dimension reaches to a threshold (e.g. 768). In this study, the time cost first increased gradually when the dimension was below 768 but tends to increase sharply (note that the $y$-axis is log-based) when the dimensionality continued to increase, as shown in Fig. 8. There, we suggest that the dimensionality should be set to approximately 768 to optimize performance and time efficiency.

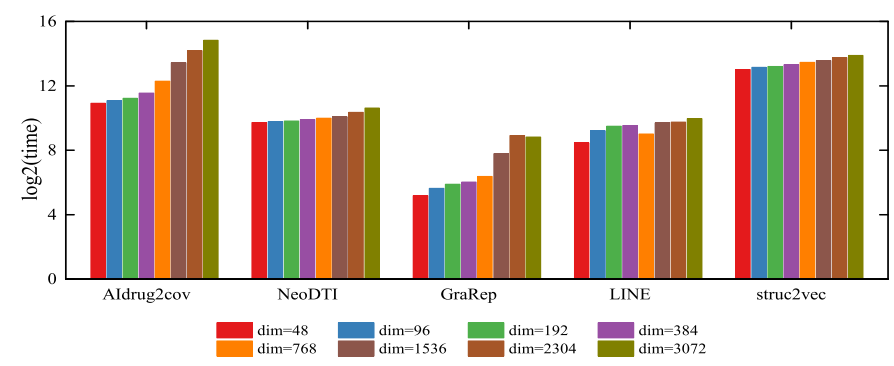

Fig. 8. Influence of dimensionality on the training time of the different embedding methods.

\section{Discussion}

In this study, we proposed AIdrug2cov, which is a network representation learning-based drug mechanism discovery and anti-inflammatory response to develop an effective therapeutic approach for COVID-19 patients. AIdrug2cov identified 40 potential targets related to COVID-19 and 25 high-confidence drugs that bind to cytokines to prevent excessive inflammatory responses in patients with COVID19. Importantly, AIdrug2cov indicated that CQ and HCQ are able to reduce fatality of COVID-19 patients, and suggested that mechanisms of action of these drugs are likely mediated through its inhibition of inflammatory cytokines on top of its antiviral ability. In addition, the results of DDI network reconstruction, ATC classification, and bio-link prediction demonstrate that AIdrug2cov significantly outperforms than other state-of-the-art network representation approaches. In summary, AIdrug2cov is a practically useful tool for accelerating COVID-19 therapeutic development.

Previous evidence reported in the literature suggests that the most popular targets predicted by AIdrug2cov are mediators of inflammation; these findings indicate that SARS-CoV-2 infection is associated with a cytokine storm and severe pulmonary inflammation in patients, consistent with the findings of several previous studies [1]. Similar to the case in patients with SARS-CoV infection, the levels of TNF- $\alpha$ [1], [21], IL1B [22], IL-6 [23], [24], CCL2 [22], [25], IFNG [27], and CXCL10 [1], [22] are significantly elevated and are associated with adverse clinical outcomes in patients with COVID-19. Therefore, this work proposed that using TNF and IL-6 target discovers high-confidence drugs for preventing cytokine storm and excessive inflammatory responses in patients with COVID-19. Recent studies have similarly suggested that drugs targeting IL-6 and TNF$\alpha$ are effective in blocking inflammatory storms, and are promising treatments for severe COVID-19 patients [1], [21], [23], [24]. The data presented in this study and the previous reports in the literature indicate that it is important to identify proteins related to COVID-19, especially those related to inflammatory response, and to apply this knowledge towards discovering candidate drugs to reduces fatality of patients with COVID-19.

In this study, we found that 7 drugs currently undergoing clinical trials for COVID-19 are able to inhibit the levels of TNF and IL-6 to reduce inflammatory response in patients with COVID-19. However, previous reports mainly focus on the antiviral effects of these drugs. In particular, we observed that CQ and HCQ affect more anti-inflammatory response pathways than antiviral pathways, indicating that CQ and HCQ for COVID-19 patients works more likely as anti-inflammatory agents than as direct antiviral agents. Interestingly, a clinical study including 550 critically ill 
COVID-19 patients has demonstrated that low dose HCQ significantly reduces fatality of critically ill COVID-19 patients, and their mechanism of action is likely mediated through its inhibition of inflammatory cytokines on top of its ability in inhibiting viral replication [16]. Meanwhile, we noticed that high concentrations of HCQ or CQ have in vitro antiviral effect on several viruses, including SARSCoV and SARS-CoV-2 [128], [129], which may contribute directly to the therapeutic effects of these drugs in COVID19 patients. Notably, we also noticed a few negative reports about CQ and HCQ [130], [131]. However, the negative results of these studies were very likely due to the use of high doses of HCQ that reduced cardiotoxicity. These studie might also have used high-dose HCQ as an antiviral agent rather than as an anti-inflammatory agent. In contrast, the dose of HCQ in [16] was much lower than that in [130], [131] but is the same as treating inflammatory diseases. Therefore, the mechanisms of action of CQ and HCQ in COVID-19 patients are likely mediated through inhibition of inflammatory cytokines on top of their antiviral effects.

On three types of pharmacological tasks, DDI network reconstruction, ATC classification, and bio-link prediction, AIdrug2cov significantly outperformed other state-of-theart network representation approaches. A major reason for the success of AIdrug2cov is that it takes into consideration types of various semantic information that indicate the anatomical therapeutic chemical of drugs to a certain extent. Recent studies have shown that the semantics of nodes are critical for knowledge discovery in real world biomedical problems [132]. Compared to previous pathbased approaches, AIdrug2cov can capture long-range dependencies without regard to their distances in the input or output sequences by relying entirely on an attention mechanism to improve representation performance.

However, we acknowledge several limitations in our current study. The top- $k$ targets and agents in this study were regarded as candidate entities related to COVID-19 according to a confidence score. The operation is simple and is popularly applied to the recommended systems, but the results neglect statistical significance to a certain extent. How to select associated candidate entities is also an important question for drug repositioning. The selection strategy of candidates must be improved in order to promote the precision of drug repositioning. For example, the confidence score could be converted to a $\mathrm{z}$-score based on permutation tests, and the corresponding $p$-value could be calculated. For each virus, those predictions with a $p$-value $<0.05$ could be treated as candidates [133].

Owing to the lack of wet-lab validation, the mechanisms of action of the COVID-19 targets and drugs could not be verified in this study. Although previous literatures provide certain evidence proving that the majority of proteins and drugs predicted by AIdrug2cov are able to target COVID19 through multiple pathways, the previous studies used different experimental platforms and viruses. However, there are some differences in mechanisms of action between different drugs and different types of cells. Therefore, standard assays must be carried out to measure the effects of these targets and drugs on the cytotoxicity, virus yield and infection rate of SARS-CoV-2, and all predicted targets and drugs must be validated in preclinical models experiments and randomized clinical trials before being used in patients.

In conclusion, this study offers a powerful network representation approach for drug mechanism discovery and anti-inflammatory response mechanism analysis that can be used to identify effective therapeutic strategies for patients with COVID-19. Our approach can increase clinical testing accuracy, which is a critical for the rapid development of efficient treatment strategies for the emerging disease COVID19. Meanwhile, the proposed AIdrug2cov method could also be applied to develop effective treatment strategies for other types of viral infections and human diseases.

\section{Method}

\subsection{Construction of a heterogeneous network}

A heterogeneous information network is defined as $G=$ $(V, E)$ where $V$ represents the set of vertices, and $E$ is the set of edges. In a heterogeneous network, each vertex $v$ and each edge $e$ are associated with an object type mapping function, $\phi(v): V \rightarrow A$, and a link type mapping function, $\psi(e): E \rightarrow R$, respectively. $A$ and $R$ denote the sets of object and link types, where $|A|+|R|>2$.

In this study, we assembled four types of nodes (i.e., drug, target, side-effect, and disease), and six types of links(i.e., drug-disease association (DDA), drug-drug interaction (DDI), drug-target interaction (DTI), drug-side-effect association (DSA), protein-protein interaction, and diseasetarget association (DisTA)) form the public databases, as shown in Fig.1(A). The drug-drug and drug-target interactions were extracted from DrugBank and ChEMBL. The human protein-protein interactions were extracted from the Human Protein Reference Database (HPRD), the Human Reference Interactome (HuRI) database and the Biological General Repository for Interaction Datasets (BioGRID). The protein-disease and drug-disease network were collected from the Toxicogenomics Database and repoDB. We also extracted the drug-side-effect associations from the Comparative Toxicogenomics Database (CTD) and the Side Effect Resource (SIDER). In addition, a protein sequence similarity network was obtained by calculating the Smith-Waterman similarities [134] of the amino acid sequence derived from UniProt. Furthermore, the drug similarity network was obtained by calculating the Tanimoto coefficient [135] from the Morgan fingerprint with a radius of 2 using the RDKit [136]. In the heterogeneous network, there were 13,619 nodes and 1,895,445 edges; all edges were unweighted, nonnegative and undirected.

\subsection{Semantic-path and deep bidirectional Transformer encoder-based network representation}

We developed a promising heterogeneous network representation approach, AIdrug2cov, by integrating semantic paths and a multi-layer bidirectional Transformer encoder model, as shown in Fig.1(B). In this work, network vertices were regarded as the vocabulary, and a set of semantic paths was treated as a corpus that is fed into a deep bidirectional Transformer encoder model to learn the lowdimensional representation of the network vertices, which existed in a continuous vector space. The proposed network representation was able to capture structural and semantical 
correlations between diverse vertices in the heterogeneous network, thus encoding latent forms of nodes.

\subsubsection{Semantic path}

A meta-path is a composite relation denoting a sequence of adjacent links between any two nodes in a heterogeneous network. The different adjacent links indicate distinct semantics. For example, meta-path(A):drug $\stackrel{\text { treats }}{\longrightarrow}$ disease $\stackrel{\text { treated by }}{\longrightarrow}$ drug represents that a disease can be treated by two drugs. Meta-path(B): drug $\stackrel{\text { binds to }}{\longrightarrow}$ protein $\stackrel{\text { causes }}{\longrightarrow}$ disease indicates that a drug binds to a protein that causes a disease. Therefore, we generated a total of 23 different meta-paths, for which detailed types of semantic path are provided in Table 5. It is noteworthy that all the semantic links in the work are reversible, and the semantic paths with lengths no longer than four. Because previous studies have indicated that meta paths with lengths greater than four may be too long to contribute to prediction [137]. In addition, Sun et al. have suggested that short meta paths are good enough, and that long meta paths may even reduce the quality of the results [138]. AIdrug2cov constructs a corpus composed of 3 billion semantic-paths that reflect the unique interaction mechanisms and topological structures among vertices in heterogeneous networks.

\subsubsection{Deep Bidirectional Transformers Encoder}

The network embedding model in AIdrug2cov is a deep bidirectional Transformer encoder based on the original implementation described in [139], and the implementation is almost identical to the original. The encoder is composed of a stack of identical layers, and every layer includes two sub-layers as shown in Fig.9. The first is a multi-head selfattention mechanism, and the second is a simple, position wise fully connected feed-forward network. In the encoder model, a residual connection [140] is employed to connect each of two sub-layers, and layer normalization is then performed.

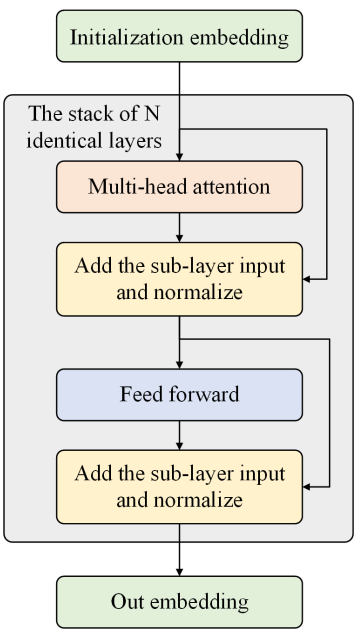

Fig. 9. Learning architecture of the deep bidirectional Transformers encoder model

\subsubsection{Training regime}

The network embedding model uses masked language learning to enable trained deep bidirectional representation
TABLE 5

The semantic path types and statistics

\begin{tabular}{|c|c|c|c|}
\hline NO. & Semantic path & & Count \\
\hline 1 & drug $\stackrel{\text { binds to }}{\longrightarrow}$ protein & & 1,923 \\
\hline 2 & drug $\stackrel{\text { binds to }}{\longrightarrow}$ protein & $\stackrel{\text { binds to }}{\longrightarrow}$ drug & 153,186 \\
\hline 3 & drug $\stackrel{\text { binds to }}{\longrightarrow}$ protein & $\stackrel{\text { interacts with }}{\longrightarrow}$ protein & 8,728 \\
\hline 4 & drug $\stackrel{\text { binds to }}{\longrightarrow}$ protein & $\stackrel{\text { causes }}{\longrightarrow}$ disease & $2,209,742$ \\
\hline 5 & drug $\stackrel{\text { binds to }}{\longrightarrow}$ protein & $\stackrel{\text { interacts with }}{\longrightarrow}$ protein $\stackrel{\text { binds to }}{\longrightarrow}$ drug & 12,734 \\
\hline 6 & drug $\stackrel{\text { binds to }}{\longrightarrow}$ protein & $\stackrel{\text { interacts with }}{\longrightarrow}$ protein $\stackrel{\text { causes }}{\longrightarrow}$ disease & $11,603,240$ \\
\hline 7 & drug $\stackrel{\text { binds to }}{\longrightarrow}$ protein & $\stackrel{\text { binds to }}{\longrightarrow}$ drug $\stackrel{\text { binds to }}{\longrightarrow}$ protein & 221,020 \\
\hline 8 & drug $\stackrel{\text { binds to }}{\longrightarrow}$ protein & $\stackrel{\text { binds to }}{\longrightarrow}$ drug $\stackrel{\text { treats }}{\longrightarrow}$ disease & $8,243,362$ \\
\hline 9 & drug $\stackrel{\text { binds to }}{\longrightarrow}$ protein & $\stackrel{\text { binds to }}{\longrightarrow}$ drug $\stackrel{\text { causes }}{\longrightarrow}$ side-effect & $4,482,541$ \\
\hline 10 & drug $\stackrel{\text { binds to }}{\longrightarrow}$ protein & $\stackrel{\text { causes }}{\longrightarrow}$ disease $\stackrel{\text { caused by }}{\longrightarrow}$ protein & $2,020,665,247$ \\
\hline 11 & drug $\stackrel{\text { binds to }}{\longrightarrow}$ protein & $\stackrel{\text { causes }}{\longrightarrow}$ disease $\stackrel{\text { treated by }}{\longrightarrow}$ drug & $231,785,524$ \\
\hline 12 & protein $\stackrel{\text { binds to }}{\longrightarrow}$ drug & $\stackrel{\text { interacts with }}{\longrightarrow}$ drug & 34,260 \\
\hline 13 & protein $\stackrel{\text { binds to }}{\longrightarrow}$ drug & $\stackrel{\text { binds to }}{\longrightarrow}$ protein & 6,344 \\
\hline 14 & protein $\stackrel{\text { binds to }}{\longrightarrow}$ drug & $\stackrel{\text { treat }}{\longrightarrow}$ disease & 636,903 \\
\hline 15 & protein $\stackrel{\text { binds to }}{\longrightarrow}$ drug & $\stackrel{\text { causes }}{\longrightarrow}$ side-effect & 270,234 \\
\hline 16 & protein $\stackrel{\text { binds to }}{\longrightarrow}$ drug & $\stackrel{\text { interacts with }}{\longrightarrow}$ drug $\stackrel{\text { binds to }}{\longrightarrow}$ protein & 60,096 \\
\hline 17 & protein $\stackrel{\text { binds to }}{\longrightarrow}$ drug & $\stackrel{\text { interacts to }}{\longrightarrow}$ drug $\stackrel{\text { treats }}{\longrightarrow}$ disease & $11,188,449$ \\
\hline 18 & protein $\stackrel{\text { binds to }}{\longrightarrow}$ drug & $\stackrel{\text { interacts with }}{\longrightarrow}$ drug $\stackrel{\text { causes }}{\longrightarrow}$ side-effect & $5,315,270$ \\
\hline 19 & protein $\stackrel{\text { binds to }}{\longrightarrow}$ drug & $\stackrel{\text { binds to }}{\longrightarrow}$ protein $\stackrel{\text { interacts with }}{\longrightarrow}$ protein & 19,371 \\
\hline 20 & protein $\stackrel{\text { binds to }}{\longrightarrow}$ drug & $\stackrel{\text { binds to }}{\longrightarrow}$ protein $\stackrel{\text { causes }}{\longrightarrow}$ disease & $13,232,097$ \\
\hline 21 & protein $\stackrel{\text { binds to }}{\longrightarrow}$ drug & $\stackrel{\text { treats }}{\longrightarrow}$ disease $\stackrel{\text { caused by }}{\longrightarrow}$ protein & $558,541,026$ \\
\hline 22 & protein $\stackrel{\text { binds to }}{\longrightarrow}$ drug & $\stackrel{\text { treats }}{\longrightarrow}$ disease $\stackrel{\text { treated by }}{\longrightarrow}$ drug & $115,924,998$ \\
\hline 23 & protein $\stackrel{\text { binds to }}{\longrightarrow}$ drug & $\stackrel{\text { causes }}{\longrightarrow}$ side-effect $\stackrel{\text { caused by }}{\longrightarrow}$ drug & $38,577,295$ \\
\hline \multicolumn{3}{|c|}{ Total number } & $3,023,193,590$ \\
\hline
\end{tabular}

inspired by the Cloze task. In masked language learning, the input corpus is randomly masked by some token, and the objective is to predict the masked word based only on its context. AIdrug2cov follows the method used in BERT [125] to mask an input corpus. First, $15 \%$ of tokens were randomly selected for masking. For every selected token, it has $80 \%$ time to be replaced by $\langle$ MASK〉 token. With $10 \%$ and $10 \%$ time, it will be randomly replaced by any other token in the dictionary or kept unchanged correspondingly. The advantage of this procedure is that the randomness can increase the generalization ability of the model, and prevent it over-fitting. In addition, because random replacement occurs is only $1.5 \%$ (i.e., $15 \%{ }^{*} 10 \%$ ) of the time for all tokens, it does not seem to harm the model's language understanding capability.

\subsection{Identification of potential targets and drugs for the COVID-19}

Based on over representations, the IMC model and CMap were used for target identification and drug repurposing for COVID-19 to facilitate therapeutic efficiency.

\subsubsection{COVID-19 target identification}

In this study, COVID-19 target identification of COVID-19 was conducted with SARS data caused by the virus SARS$\mathrm{CoV}$ since SARS-CoV and SARS-CoV-2 are highly similar and closely related coronaviruses. Phylogenetic analysis has 
revealed that the genome of SARS-CoV-2 is similar to that of SARS-CoV, with approximately $79 \%$ sequence identity [4]. Further sequence alignment has revealed that the similarity of the sequence of the main protease between SARS-CoV-2 and SARS-CoV, which is essential for the life cycle of the virus, is up to $96.1 \%$. In addition, the pathogenic mechanisms of SARS-CoV-2 and SARS-CoV are highly similar [3]. Therefore, predicted targets related to SARS can reasonably be treated as potential targets of COVID-19.

As shown in Fig.1(E), in the process of COVID-19 target identification, the low-dimensional vector of nodes was feed into the IMC model to predict the confidence scores of SARS-protein interactions. Then, the proteins were ranked according to confidence scores, and the top- $k$ proteins were regard as potential targets of COVID-19. Finally, Enrichr [18], a comprehensive gene set enrichment analysis tool, was used to perform functional enrichment analyse, including GO and KEGG enrichment analyses to evaluate the functional pathways and biological relevance of the potential targets of COVID-19.

\subsubsection{Anti-inflammatory drug discovery for COVID-19}

Studies have shown that SARS-CoV-2 induces excessive and aberrant non-effective host immune responses that are associated with severe lung pathology and lead to death [1]. Similar to SARS-CoV infection, SARS-CoV-2 infection is also associated with a cytokine storm and severe pulmonary inflammation in moribund patients, and is characterized mainly by elevated plasma concentrations of IL-6, TNF$\alpha$. In particular, researchers have proposed that IL-6, and TNF- $\alpha$ might be promising therapeutic targets. Therefore, this study predicted drugs related to IL- 6 or TNF- $\alpha$ to facilitate the therapies efficacy. Similar to the methods for target identification, IMC model are integrated to predict confidence scores for interaction between drugs and IL6 or TNF- $\alpha$ and drugs, and the top- $k$ candidate drugs according to confidence scores were selected for IL-6 or TNF- $\alpha$, respectively. In addition, we performed the CMap [19] analysis to further screen candidate drugs for COVID19 . Due to the lack of gene expression data from the SARSCoV-2 infected patients, we used the gene expression profiles of peripheral blood mononuclear cells (PBMCs) from ten SARS-CoV infected patients (GEO:GSE1739) [141] to identify potential COVID-19 therapeutic drugs. The detailed connectivity analysis steps are listed as follows.

Step 1: Student's t test was performed to identify genes that were differentially expressed in samples from SARS patients compared with normal samples. For each gene, the statistical significance was assessed by computing the $p$ value. The $\log _{2}$ (FC) value was calculated as the fold change (FC) between the average signal intensity of 10 SARS patients and that of 4 normal human subjects was calculated for each gene. Any gene meeting the criteria of a $p<0.01$ and an absolute $\log _{2}(\mathrm{FC})>1$ was considered to be the up- and down-regulated genes.

Step 2: The CMap scores were computed based on the sets of up- and down-regulated genes in SARS-CoV infected patients by using a web server (https://clue.io/query).

Step 3: In AIdrug2cov, under the hypothesis that if a drug has a gene expression signature that is opposite to a disease signature, that drug could potentially be used as a treatment for that disease [142]. Therefore, drugs with the CMap scores $<0$ were treated as COVID-19 therapeutic drug candidates.

Finally, we used literature-reported knowledge to filter drugs that tended to increase the release of IL- 6 or TNF- $\alpha$.

\subsection{Pharmacological application of Aldrug2cov}

To evaluate and interpret the node representation performance of AIdrug2cov, we performed various pharmacological tests, including DDI network reconstruction, ATC classification, and bio-link (i.e., DisTA, TDI, DSA) prediction.

\subsubsection{DDI network reconstruction}

As network representations, embedding vectors are expected to reconstruct the original networks well [126]. Here, we reconstructed the DDI network edges based on the proximity nodes to evaluate the representation performance of AIdrug2cov. First, the proximity matrix was attained by directly calculating the cosine similarity between embedding vectors. Then, the pairs of nodes were ranked according to their proximity. Finally, the ratio of real links in the top $k$ pairs of vertices was treated as the reconstruction precision. A more reconstruction precision indicated a more higher embedding quality.

\subsubsection{ATC classification}

In drug development, identification of the ATC class of an uncharacterized compound is a challenging and important task, since such a prediction system could be used to deduce not only a compound's possible active ingredients but also its chemical, therapeutic, pharmacological, and other properties.In addition, node classification, which aims to predict the classes of unlabeled nodes for a partially labeled network, is one of the most important tasks in network analysis. Therefore, the AIdrug2cov low-dimensional embedding vectors were treated as feature of nodes, and fed into the Multi-label K-Nearest Neighbor (ML-KNN) [143] model which is frequently used to predict the ATC classes of drug. Generally, good network embedding should capture the network structure and hence be useful for ATC classification.

\subsubsection{Bio-link prediction}

Another important task of network embedding is predicting unobserved links in a network, which refers to predicting either missing interactions that may appear in the future. Link prediction is pervasive in biological network analysis, but verifying the existence of links between nodes is time-consuming and cost-expensive [144]. Therefore, a great number of efforts have been devoted to predicting potential interactions based on network embedding approaches, such as deeper [145], and NeoDTI [122].

To further demonstrate the effectiveness of the proposed embedding methods, the IMC model was also employed to predict DisTA, TDI, and DSA, that is, disease $\rightarrow$ protein $\rightarrow$ drug $\rightarrow$ side-effect associations. The IMC model has been widely used for biomedical link prediction, such as drug-target interaction prediction [146], and gene-disease interaction prediction [147]. The previous findings suggest that a good network representation model can significantly improve prediction accuracy, and should be able to capture the inherent structure of a network well enough to predict likely but unobserved links. 


\section{AVAILABILITY AND IMPLEMENTATION}

The source code and data of AIdrug2cov can be downloaded from https://github.com/pengsl-lab/AIdrug2cov.git.

\section{ACKNOWLEDGMENTS}

This work was supported by National Key R\&D Program of China 2017YFB0202602, 2018YFC0910405, 2017YFC1311003, 2016YFC1302500, 2016YFB0200400, 2017YFB0202104; NSFC Grants 81973244, U19A2067, 61772543, U1435222, 61625202, 61272056; The Funds of Peng Cheng Lab, State Key Laboratory of Chemo/Biosensing and Chemometrics; the Fundamental Research Funds for the Central Universities, and Guangdong Provincial Department of Science and Technology under grant No. 2016B090918122.

\section{REFERENCES}

[1] C. Huang, Y. Wang, X. Li, L. Ren, J. Zhao, Y. Hu, L. Zhang, G. Fan, J. Xu, X. Gu et al., "Clinical features of patients infected with 2019 novel coronavirus in wuhan, china," The Lancet, vol. 395, no. 10223, pp. 497-506, 2020.

[2] S. Pushpakom, F. Iorio, P. A. Eyers, K. J. Escott, S. Hopper, A. Wells, A. J. Doig, T. Guilliams, J. Latimer, C. J. Mcnamee et al., "Drug repurposing: progress, challenges and recommendations," Nature Reviews Drug Discovery, vol. 18, no. 1, pp. 41-58, 2019.

[3] J. Xu, S. Zhao, T. Teng, A. E. Abdalla, W. Zhu, L. Xie, Y. Wang, and X. Guo, "Systematic comparison of two animal-to-human transmitted human coronaviruses: Sars-cov-2 and sars-cov," Viruses, vol. 12, no. 2, p. 244, 2020.

[4] R. Lu, X. Zhao, J. Li, P. Niu, B. Yang, H. Wu, W. Wang, H. Song, B. Huang, N. Zhu et al., "Genomic characterisation and epidemiology of 2019 novel coronavirus: implications for virus origins and receptor binding," The Lancet, vol. 395, no. 10224, pp. 565574,2020 .

[5] P. Zhou, X. Yang, X. Wang, B. Hu, L. Zhang, W. Zhang, H. Si, Y. Zhu, B. Li, C. Huang et al., "A pneumonia outbreak associated with a new coronavirus of probable bat origin," Nature, vol. 579, no. 7798, pp. 270-273, 2020.

[6] C. M. Poh, G. Carissimo, B. Wang, S. N. Amrun, C. Y. P. Lee, R. S. L. Chee, S. W. Fong, N. K. W. Yeo, W. H. Lee, R. A. Torres, Y. S. Leo, M. I. C. Chen, S. Y. Tan, L. Y. A. Chai, S. Kalimuddin, S. S. G. Kheng, S. Y. Thien, B. E. Young, D. C. Lye, B. J. Hanson, C. I. Wang, L. Renia, and L. F. Ng, "Two linear epitopes on the sars-cov-2 spike protein that elicit neutralising antibodies in covid-19 patients," Nature Communications, 2020.

[7] X. Y. Ou, Y. Liu, X. B. Lei, P. Li, D. Mi, L. Ren, L. Guo, R. X. Guo, T. Chen, J. X. Hu, Z. C. Xiang, Z. X. Mu, X. Chen, J. Y. Chen, K. P. $\mathrm{Hu}, \mathrm{Q}$. Jin, J. W. Wang, and Z. H. Qian, "Characterization of spike glycoprotein of sars-cov-2 on virus entry and its immune crossreactivity with sars-cov," Nature Communications, vol. 11, no. 1, p. 1620, 2020.

[8] M. Letko and V. J. Munster, "Functional assessment of cell entry and receptor usage for lineage $\mathrm{b}$-coronaviruses, including 2019ncov," bioRxiv, 2020.

[9] Y. Zhang and T. G. Kutateladze, "Molecular structure analyses suggest strategies to therapeutically target sars-cov-2," Nature Communications, 2020.

[10] T. R. F. Smith, A. Patel, S. Ramos, D. Elwood, X. Zhu, J. Yan, E. N. Gary, S. N. Walker, K. Schultheis, M. Purwar, Z. Xu, J. Walters, P. Bhojnagarwala, M. Yang, N. Chokkalingam, P. Pezzoli, E. Parzych, E. L. Reuschel, A. Doan, N. Tursi, M. Vasquez, J. Choi, E. Tello-Ruiz, I. Maricic, M. A. Bah, Y. Wu, D. Amante, D. H. Park, Y. Dia, A. R. Ali, F. I. Zaidi, A. Generotti, K. Y. Kim, T. A. Herring, S. Reeder, V. M. Andrade, K. Buttigieg, G. Zhao, J. Wu, D. Li, L. Bao, J. Liu, W. Deng, C. Qin, A. S. Brown, M. Khoshnejad, N. Wang, J. Chu, D. Wrapp, J. S. McLellan, K. Muthumani, B. Wang, M. W. Carroll, J. J. Kim, J. Boyer, D. W. Kulp, L. M. Humeau, D. B. Weiner, and K. E. Broderick, "Immunogenicity of a dna vaccine candidate for covid-19," Nature Communications, 2020.
[11] M. Merad and J. C. Martin, "Pathological inflammation in patients with covid-19: a key role for monocytes and macrophages," Nature reviews immunology, vol. 20, no. 6, pp. 355-362, 2020.

[12] P. Mehta, D. F. Mcauley, M. Brown, E. Sanchez, R. Tattersall, and J. Manson, "Covid-19: consider cytokine storm syndromes and immunosuppression," The Lancet, 2020.

[13] C. Chengshui, Q. Feng, S. Keqing, L. Yuping, L. Ji, C. Yongping, P. Jingye, Z. Tieli, L. Xiangyang, Z. Jinsan, L. Yongde, L. Xiaokun, and X. Jinglin, "Thalidomide combined with low-dose glucocorticoid in the treatment of covid-19 pneumonia," Preprints, 2020.

[14] F. X. Cheng, R. J. Desai, D. E. Handy, R. S. Wang, S. SchneeweisS, A. Barabasi, and J. Loscalzo, "Network-based approach to prediction and population-based validation of in silico drug repurposing." Nature Communications, vol. 9, no. 1, pp. 2691-2691, 2018.

[15] F. X. Cheng, I. Kovacs, and A. Barabasi, "Network-based prediction of drug combinations," Nature Communications, vol. 10, no. 1, p. 1197, 2019.

[16] B. Yu, C. Li, P. Chen, N. Zhou, L. Wang, J. Li, H. Jiang, and D. Wang, "Low dose of hydroxychloroquine reduces fatality of critically ill patients with covid-19," Science China Life Sciences, 2020.

[17] P. Jain and I. S. Dhillon, "Provable inductive matrix completion," arXiv preprint, 2013.

[18] E. Y. Chen, C. M. Tan, Y. Kou, Q. N. Duan, Z. C. Wang, G. V. Meirelles, N. R. Clark, and A. Maayan, "Enrichr: interactive and collaborative html5 gene list enrichment analysis tool," BMC Bioinformatics, vol. 14, no. 1, pp. 128-128, 2013.

[19] J. Lamb, E. D. Crawford, D. Peck, J. W. Modell, I. C. Blat, M. J. Wrobel, J. Lerner, J. Brunet, A. Subramanian, K. N. Ross et al., "The connectivity map: Using gene-expression signatures to connect small molecules, genes, and disease," Science, vol. 313, no. 5795, pp. 1929-1935, 2006.

[20] P. T. Lang, S. R. Brozell, S. Mukherjee, E. F. Pettersen, E. C. Meng, V. Thomas, R. C. Rizzo, D. A. Case, T. L. James, and I. D. Kuntz, "Dock 6: Combining techniques to model rnacsmall molecule complexes," RNA, vol. 15, no. 6, pp. 1219-1230, 2009.

[21] M. Feldmann, R. Maini, J. Woody, S. Holgate, G. Winter, M. Rowland, D. Richards, and T. Hussell, "Trials of anti-tumour necrosis factor therapy for covid-19 are urgently needed," The Lancet, vol. 395, no. 10234, pp. 1407-1409, 2020.

[22] M. Merad and J. C. Martin, "Pathological inflammation in patients with covid-19: a key role for monocytes and macrophages," Nature Reviews Immunology, 2020.

[23] J. B. Moore1 and C. H. June, "Cytokine release syndrome in severe covid-19," Science, vol. 368, no. 6490, p. eabb8925, 2020.

[24] X. H. Chen, B. H. Zhao, Y. M. Qu, Y. R. Chen, J. Xiong, Y. Feng, D. Men, Q. C. Huang, Y. Liu, B. Yang, J. Y. Ding, and L. F, "Detectable serum sars-cov-2 viral load (rnaaemia) is closely correlated with drastically elevated interleukin 6 (il-6) level in critically ill covid-19 patients," The Lancet, 2020.

[25] S. Wan, Q. Yi, S. B. Fan, J. Lv, X. X. Zhang, L. Guo, C. H. Lang Q. Xiao, K. Xiao, Z. Yi, Q. Mao, J. L. Xiang, B. S. Zhang, and C. Y. $\mathrm{P}$, "Characteristics of lymphocyte subsets and cytokines in peripheral blood of 123 hospitalized patients with 2019 novel coronavirus pneumonia (ncp)," medRxiv, 2020.

[26] C. Qin, L. Q. Zhou, Z. W. Hu, S. Q. Zhang, S. Yang, Y. Tao, C. H. Xie, K. Ma, K. Shang, W. Wang, and D.-S. Tian, "Dysregulation of immune response in patients with covid-19 in wuhan, china," Clinical Infectious Diseases, 2020.

[27] N. S. Chen, M. Zhou, X. Dong, J. M. Qu, F. Y. Gong, Y. Han Y. Qiu, J. L. Wang, Y. Liu, Y. Wei, J. A. Xia, T. Yu, X. X. Zhang, and L. Zhang, "Epidemiological and clinical characteristics of 99 cases of 2019 novel coronavirus pneumonia in wuhan, china: a descriptive study," The Lancet, vol. 395, no. 10223, pp. 507-513, 2020.

[28] T. P. Velavan and C. G. Meyer, "The covid-19 epidemic," Tropical Medicine and International Health, vol. 25, no. 3, pp. 278-280, 2020.

[29] Y. Malauer, J. Carbajolozoya, M. Y. Hein, M. A. Muller, W. Deng, J. Lei, B. Meyer, Y. Kusov, B. Von Brunn, D. R. Bairad et al., "p53 down-regulates sars coronavirus replication and is targeted by the sars-unique domain and plpro via e3 ubiquitin ligase rchy1," Proceedings of the National Academy of Sciences of the United States of America, vol. 113, no. 35, p. 201603435, 2016.

[30] R. Gill, S. Frederick, C. B. Hartline, S. Chou, and M. N. Prichard, "Conserved retinoblastoma protein-binding motif in human cytomegalovirus ul97 kinase minimally impacts viral replication 
but affects susceptibility to maribavir." Virology Journal, vol. 6, no. 1, pp. 9-9, 2009.

[31] H. Sobhy, "A review of functional motifs utilized by viruses," Proteome, vol. 4, no. 1, p. 3, 2016.

[32] Z. D. Ye, C. K. Wong, P. Li, and Y. Xie, "A sars-cov protein, orf-6, induces caspase- 3 mediated, er stress and jnk-dependent apoptosis," Biochimica et Biophysica Acta, vol. 1780, no. 12, pp. 1383-1387, 2008.

[33] T. Mizutani, S. Fukushi, M. Saijo, I. Kurane, and S. Morikawa, "Phosphorylation of p38 mapk and its downstream targets in sars coronavirus-infected cells," Biochemical and Biophysical Research Communications, vol. 319, no. 4, pp. 1228-1234, 2004.

[34] C. M. Chan, C. W. Ma, W. Y. Chan, and H. Y. E. Chan, "The sars-coronavirus membrane protein induces apoptosis through modulating the akt survival pathway," Archives of Biochemistry and Biophysics, vol. 459, no. 2, pp. 197-207, 2007.

[35] Y. S. Yeung, C. W. Yip, C. C. Hon, K. Y. C. Chow, I. C. M. Ma, F. Y. Zeng, and F. C. Leung, "Transcriptional profiling of vero e6 cells over-expressing sars-cov s2 subunit: Insights on viral regulation of apoptosis and proliferation," Virology, vol. 371, no. 1, pp. 3243, 2008.

[36] T. Mizutani, S. Fukushi, M. Saijo, I. Kurane, and S. Morikawa, "Jnk and pi3k/akt signaling pathways are required for establishing persistent sars-cov infection in vero e6 cells," Biochimica et Biophysica Acta, vol. 1741, no. 1, pp. 4-10, 2005.

[37] C. W, "A potential treatment of covid-19 with tgf- blockade," International Journal of Biological Sciences, vol. 16, no. 11, pp. 19541955, 2020.

[38] C. Y. Wang, C. Y. Lu, S. W. Li, C. C. Lai, C. H. Hua, S. H. Huang, Y. J. Lin, M. J. Hour, and C. W. Lin, "Sars coronavirus papain-like protease up-regulates the collagen expression through non-samd tgf-1 signaling," Virus Research, vol. 235, pp. 58-66, 2017.

[39] T. Venkataraman and M. B. Frieman, "The role of epidermal growth factor receptor (egfr) signaling in sars coronavirusinduced pulmonary fibrosis," Antiviral Research, vol. 143, pp. 142 150, 2017.

[40] M. A. Lari, "An evidence for correlation between the glutathione s-transferase t1 (gstt1) polymorphism and outcome of covid-19," Clinica Chimica Acta, vol. 58, pp. 213-216, 2020.

[41] D. Thomas-Rddel, J.Winning, P. Dickmann, D. Ouart, and A. Kortgen1, "Coronavirus disease 2019 (covid-19): Update for anesthesiologists and intensivists march 2020," Anaesthesist, vol. 69, pp. 225-235, 2020.

[42] M. Turkia, "Covid-19 as an endothelial disease and its relationship to vascular endothelial growth factor (vegf) and iodide," SSRN Electronic Journal, 2020.

[43] N. Stefan, A. L. Birkenfeld, M. B. Schulze, and D. S. Ludwig, "Obesity and impaired metabolic health in patients with covid19," Nature Reviews Endocrinology, 2020.

[44] D. Blanco-Melo, B. E. Nilsson-Payant, W. C. Liu, S. Uhl, D. Hoagland, R. M $\phi$ ller, T. Jordan, K. Oishi, M. Panis, D. Sachs, T. Wang, R. Schwartz, J. K. Lim, R. A. Albrecht, and B. R. tenOever, "Imbalanced host response to sars-cov-2 drives development of covid-19," Cell, vol. 181, no. 5, pp. 1036-1045, 2020.

[45] S. Kumar, "Covid-19: A drug repurposing and biomarker identification by using comprehensive gene-disease associations through protein-protein interaction network analysis," Preprints, 2020.

[46] M. Surjit and S. K. Lal, “The sars-cov nucleocapsid protein: a protein with multifarious activities." Infection, Genetics and Evolution, vol. 8, no. 4, pp. 397-405, 2008.

[47] S. M, L. B, C. VT, and L. SK, "The nucleocapsid protein of severe acute respiratory syndrome-coronavirus inhibits the activity of cyclin-cyclin-dependent kinase complex and blocks s phase progression in mammalian cells," journal of biological chemistry, vol. 281, no. 16, pp. 10669-81, 2006.

[48] M. L. Dediego, J. L. Nietotorres, J. M. Jimenezguardeno, J. A Reglanava, C. Castanorodriguez, R. Fernandezdelgado, F. Usera, and L. Enjuanes, "Coronavirus virulence genes with main focus on sars-cov envelope gene," Virus Research, vol. 194, pp. 124-137, 2014.

[49] M. Gharote, "Role of parp-1 inhibition by nicotinamide to modulate host immune response and prevention of cytokine storm in covid-19," Indian Journal of Medical Sciences, vol. 72, pp. 21-24, 2020.

[50] F. M. Konrad, U. Knausberg, R. Hone, K. Ngamsri, and J. Reutershan, "Tissue heme oxygenase- 1 exerts anti-inflammatory effects on lps-induced pulmonary inflammation," Mucosal Immunology, vol. 9, no. 1, pp. 98-111, 2016

[51] J. A. Espinoza, P. A. Gonzalez, and A. M. Kalergis, "Modulation of antiviral immunity by heme oxygenase-1," American Journal of Pathology, vol. 187, no. 3, pp. 487-0493, 2017.

[52] W. Y. Dong, W. T. Xie, Y. B. Liu, B. K. Sui, H. Zhang, L. Liu, Y. Tan, X. H. Tong, Z. F. Fu, and P. Yin, "Receptor tyrosine kinase inhibitors block proliferation of tgev mainly through p38 mitogen-activated protein kinase pathways," Antiviral Research, vol. 173, p. 104651, 2020.

[53] S. Kumar, J. C. Boehm, and J. C. Lee, "p38 map kinases: key signalling molecules as therapeutic targets for inflammatory diseases," Nature Reviews Drug Discovery, vol. 2, no. 9, pp. 717-726, 2003.

[54] R. Gnoni, Martin; Vasquez-Gararatti and A. Mena, "Possible role of tetracyclines on covid-19: Recycling well-known old drugs from the shelf," The University of Louisville Journal of Respiratory Infections, vol. 4, 2020.

[55] H. H. Ng, T. Narasaraju, M. C. Phoon, M. Sim, J. E. Seet, and V. T. K. Chow, "Doxycycline treatment attenuates acute lung injury in mice infected with virulent influenza h3n2 virus: Involvement of matrix metalloproteinases," Experimental and Molecular Pathology, vol. 92, no. 3, pp. 287-295, 2012.

[56] R. Channappanavar and S. Perlman, "Pathogenic human coronavirus infections: causes and consequences of cytokine storm and immunopathology," Seminars in Immunopathology, vol. 39, no. 5, pp. 529-539, 2017.

[57] G. A. Fitzgerald, "Misguided drug advice for covid-19," Science, 2020.

[58] J. Ouyang, J. Hu, and J. Chen, "Incrnas regulate the innate immune response to viral infection," Wiley Interdisciplinary ReviewsRna, vol. 7, no. 1, pp. 129-143, 2016.

[59] X. H. Yan, Q. Hao, Y. X. Mu, K. Timani, L. B. Ye, Y. Zhu, and W. J. $\mathrm{G}$, "Nucleocapsid protein of sars-cov activates the expression of cyclooxygenase- 2 by binding directly to regulatory elements for nuclear factor-kappa $\mathrm{b}$ and ccaat/enhancer binding protein," The International Journal of Biochemistry and Cell Biology, vol. 38, no. 8, pp. 1417-28, 2006.

[60] T. Mizutani, S. Fukushi, K. Ishii, Y. Sasaki, T. Kenri, M. Saijo, Y. Kanaji, K. Shirota, I. Kurane, and S. Morikawa, "Mechanisms of establishment of persistent sars-cov-infected cells," Biochemical and Biophysical Research Communications, vol. 347, no. 1, pp. 261$265,2006$.

[61] I. Darwish, S. Mubareka, and W. C. Liles, "Immunomodulatory therapy for severe influenza," Expert Review of Anti-infective Therapy, vol. 9, no. 7, pp. 807-822, 2011.

[62] S. F. Dosch, S. D. Mahajan, and A. R. Collins, "Sars coronavirus spike protein-induced innate immune response occurs via activation of the nf-b pathway in human monocyte macrophages in vitro," Virus Research, vol. 142, no. 1, pp. 19-27, 2009.

[63] S. Akerstrom, V. Gunalan, C. T. Keng, Y. Tan, and A. Mirazimi, "Dual effect of nitric oxide on sars-cov replication: Viral rna production and palmitoylation of the s protein are affected," Virology, vol. 395, no. 1, pp. 1-9, 2009.

[64] S. Akerstrom, M. Mousavijazi, J. Klingstrom, M. Leijon, A. Lundkvist, and A. Mirazimi, "Nitric oxide inhibits the replication cycle of severe acute respiratory syndrome coronavirus," Journal of Virology, vol. 79, no. 3, pp. 1966-1969, 2005.

[65] E. J. Molloy, "Covid-19 in children and altered inflammatory responses," Pediatric Research, vol. 72, 2020.

[66] I. Chen, S. C. Chang, H. Y. Wu, T. C. Yu, W. C. Wei, S. M. Lin C. L. Chien, and M. F. Chang, "Upregulation of the chemokine (c-c motif) ligand 2 via a severe acute respiratory syndrome coronavirus spike-ace2 signaling pathway," Journal of Virology, vol. 84, no. 15, pp. 7703-7712, 2010.

[67] S. L. Yan, S. Wu, M. Yin, H. T. Chen, and H. Chen, "Protective effects from carnosine and histidine on acetaminophen-induced liver injury," Journal of Food Science, vol. 74, no. 8, 2009.

[68] M. Bourdi, Y. Masubuchi, T. P. Reilly, H. R. Amouzadeh, J. L. Martin, J. W. George, A. G. Shah, and L. R. Pohl, "Protection against acetaminophen-induced liver injury and lethality by interleukin 10: Role of inducible nitric oxide synthase," Hepatology, vol. 35, no. 2, pp. 289-298, 2002.

[69] N. Yang, Y. Liang, P. Yang, W. Wang, X. Zhang, and J. Wang, "Tnf$\alpha$ receptor antagonist attenuates isoflurane-induced cognitive impairment in aged rats," Experimental and Therapeutic Medicine, vol. 12, no. 1, pp. 463-468, 2016. 
[70] K. Zhang, P. P. Zhao, G. Y. Guo, Y. Guo, L. Tian, X. Sun, S. W. Li, Y. He, Y. Sun, H. L. Chai, W. Zhang, and M. W. Xing, "Arsenic trioxide attenuates nf-b and cytokine mrna levels in the livers of cocks," Biological Trace Element Research, vol. 170, no. 2, pp. 432437, 2016.

[71] G. M. Thiele, G. E. Hill, J. A. Pavlik, T. L. Freeman, D. J. Tuma, M. J. Duryee, and L. W. Klassen, "Halothane potentiates the alcohol-adduct induced tnf-alpha release in heart endothelial cells," BMC Anesthesiology, vol. 5, no. 1, pp. 3-3, 2005.

[72] Y. Liu, Y. Liu, D. Xu, and J. Li, "Latanoprost-induced cytokine and chemokine release from human tenon's capsule fibroblasts: Role of mapk and nf-b signaling pathways." Journal of Glaucoma, vol. 24, no. 9, pp. 635-641, 2015.

[73] Q. Z. Li, Q. Deng, J. Q. Li, G. H. Yi, and s. P. Zhao, "Valsartan reduces interleukin-1 $\beta$ secretion by peripheral blood mononuclear cells in patients with essential hypertension," Clinica chimica acta, vol. 355, pp. 131-136, 2005.

[74] A. L. Moreira, E. P. Sampaio, A. Zmuidzinas, P. Frindt, K. A. Smith, and G. Kaplan, "Thalidomide exerts its inhibitory action on tumor necrosis factor alpha by enhancing mrna degradation." Journal of Experimental Medicine, vol. 177, no. 6, pp. 1675-1680, 1993.

[75] B. E. Turk, H. Jiang, and J. O. Liu, "Binding of thalidomide to alpha1-acid glycoprotein may be involved in its inhibition of tumor necrosis factor alpha production." Proceedings of the National Academy of Sciences of the United States of America, vol. 93, no. 15, pp. 7552-7556, 1996.

[76] N. Enomoto, Y. Takei, M. Hirose, K. Ikejima, H. Miwa, T. Kitamura, and N. Sato, "Thalidomide prevents alcoholic liver injury in rats through suppression of kupffer cell sensitization and tnfproduction," Gastroenterology, vol. 123, no. 1, pp. 291-300, 2002.

[77] J. L. Tavares, A. Wangoo, P. Dilworth, B. G. Marshall, S. Kotecha, and R. J. Shaw, "Thalidomide reduces tumour necrosis factor$\alpha$ production by human alveolar macrophages," Respiratory Medicine, vol. 91, no. 1, pp. 31-39, 1997.

[78] C. H. Jang, J. H. Choi, M. S. Byun, and D. Jue, "Chloroquine inhibits production of tnf- $\alpha$, il- $1 \beta$ and il-6 from lipopolysaccharide-stimulated human monocytes/macrophages by different modes," Rheumatology, vol. 45, no. 6, pp. 703-710, 2006.

[79] D. Zhou, Y. Liu, L. H. Xu, D. Y. Ouyang, H. Pan, X. Y. Zhang, G. X. Zhao, and X. H. He, "Chloroquine differentially modulates inflammatory cytokine expression in raw 264.7 cells in response to inactivatedstaphylococcus aureus," Inflammation, vol. 38, no. 2, pp. 745-755, 2015.

[80] J. Y. Jeong and D. M. Jue, "Chloroquine inhibits processing of tumor necrosis factor in lipopolysaccharide-stimulated raw 264.7 macrophages," Journal of Immunology, vol. 158, no. 10, pp. 49014907, 1997.

[81] D. Marx, M. Tassabehij, S. Heer, K. B. Huttenbrink, and I. P. Szelenyi, "Modulation of tnf and gm-csf release from dispersed human nasal polyp cells and human whole blood by inhibitors of different pde isoenzymes and glucocorticoids." Pulmonary Pharmacology and Therapeutics, vol. 15, no. 1, pp. 7-15, 2002.

[82] A. L. Gilman, F. Beams, M. Tefft, and A. Mazumder, "The effect of hydroxychloroquine on alloreactivity and its potential use for graft-versus-host disease," Bone Marrow Transplantation, vol. 17, no. 6, pp. 1069-1076, 1996.

[83] K. Sperber, H. Quraishi, T. H. Kalb, A. Panja, Stecher, and L. Mayer, "Selective regulation of cytokine secretion by hydroxychloroquine: inhibition of interleukin 1 alpha (il-1-alpha) and il-6 in human monocytes and t cells." The Journal of Rheumatology, vol. 20, no. 5, pp. 803-808, 1993.

[84] M. Wang, R. Cao, L. Zhang, X. Yang, J. Liu, M. Xu, Z. Shi, Z. Hu, W. Zhong, and G. Xiao, "Remdesivir and chloroquine effectively inhibit the recently emerged novel coronavirus (2019-ncov) in vitro," Cell Research, vol. 30, no. 3, pp. 269-271, 2020.

[85] M. J. Vincent, E. Bergeron, S. Benjannet, B. R. Erickson, P. E. Rollin, T. G. Ksiazek, N. G. Seidah, and S. T. Nichol, "Chloroquine is a potent inhibitor of sars coronavirus infection and spread," Virology Journal, vol. 2, no. 1, pp. 69-69, 2005.

[86] C. K. Fraser, E. L. Lousberg, R. Kumar, T. P. Hughes, K. R. Diener, and J. D. Hayball, "Dasatinib inhibits the secretion of tnf- following tlr stimulation in vitro and in vivo," Experimental Hematology, vol. 37, no. 12, pp. 1435-1444, 2009.

[87] Q. Zhang, X. Xiao, M. Li, W. Li, M. Yu, H. Zhang, Z. Wang, and H. Xiang, "Acarbose reduces blood glucose by activating mir- 10a-5p and mir-664 in diabetic rats," PLOS ONE, vol. 8, no. 11, 2013.

[88] A. L. Wang, A. C. H. Yu, L. T. Lau, C. Lee, L. M. Wu, X. Zhu, and M. O. M. Tso, "Minocycline inhibits lps-induced retinal microglia activation." Neurochemistry International, vol. 47, no. 1, pp. 152 158, 2005.

[89] A. M. Wolf, D. Wolf, H. Rumpold, S. Ludwiczek, B. Enrich, G. Gastl, G. Weiss, and H. Tilg, "The kinase inhibitor imatinib mesylate inhibits tnf- production in vitro and prevents tnfdependent acute hepatic inflammation," Proceedings of the $\mathrm{Na}$ tional Academy of Sciences of the United States of America, vol. 102, no. 38, pp. 13622-13627, 2005.

[90] X. Y. Liu, , S. Liu, Z. H. Chen, Z. Y. Zhao, Y. Y. Huang, Q. L Zhang, J. Wang, Y. Shi, Y. H. Xu, J. Sun, H. F. Xian, R. L. Fang, F. Bai, C. X. Ou, B. Xiong, A. M. Lew, J. Cui, H. Hui, J. Zhao, X. C. Hong, Y. X. Zhang, F. L. Zhou, and H. B. Luo, "Therapeutic effects of dipyridamole on covid-19 patients with coagulation dysfunction," medRxiv, 2020.

[91] S. Sloka, L. M. Metz, W. Hader, Y. Starreveld, and V. W. Yong, "Reduction of microglial activity in a model of multiple sclerosis by dipyridamole," Journal of Neuroinflammation, vol. 10, no. 1, pp. 89-89, 2013

[92] A. Matsumori, Y. Nunokawa, and S. Sasayama, "Nifedipine inhibits activation of transcription factor nf-b," Life Sciences, vol. 67, no. 21, pp. 2655-2661, 2000.

[93] S. Yamagishi, Y. Inagaki, R. Abe, S. Kikuchi, N. Sasaki, and M. Takeuchi, "Nifedipine inhibits apoptotic cell death of cultured endothelial cells induced by tumor necrosis factor-alpha." Drugs Under Experimental and Clinical Research, vol. 29, no. 4, pp. 141145, 2003.

[94] C. Anna, B. Svetlana, Z. Ruslan, K. Thomas, L. Victor, N. Sergei, and D. Marina, "Effects of imiquimod treatment on tnf expression in two-step skin carcinogenesis model in mice," Frontiers in Immunology, vol. 4, 2013.

[95] E. B. Cook, J. L. Stahl, N. P. Barney, and F. M. Graziano, "Olopatadine inhibits tnf release from human conjunctival mast cells," Annals of Allergy Asthma and Immunology, vol. 84, no. 5, pp. 504508,2000

[96] J. I. Mcgill, "A review of the use of olopatadine in allergic conjunctivitis," International Ophthalmology, vol. 25, no. 3, pp. 171179, 2004.

[97] M. Baz, J. Carbonneau, C. Rheaume, M. Cavanagh, and G. Boivin, "Combination therapy with oseltamivir and favipiravir delays mortality but does not prevent oseltamivir resistance in immunodeficient mice infected with pandemic a(h1n1) influenza virus." Viruses, vol. 10, no. 11, p. 610, 2018.

[98] F. G. Hayden, J. J. Treanor, R. S. Fritz, M. Lobo, R. F. Betts, M. Miller, N. Kinnersley, R. G. Mills, P. Ward, and S. E. Straus, "Use of the oral neuraminidase inhibitor oseltamivir in experimental human influenza: Randomized controlled trials for prevention and treatment," JAMA, vol. 282, no. 13, pp. 1240-1246, 1999.

[99] J. Jimenez, J. Iribarren, L. Lorente, J. M. Rodriguez, D. Hernandez, I. Nassar, R. Perez, M. Brouard, A. Milena, R. Martinez et al. "Tranexamic acid attenuates inflammatory response in cardiopulmonary bypass surgery through blockade of fibrinolysis: a case control study followed by a randomized double-blind controlled trial," Critical Care, vol. 11, no. 6, pp. 1-10, 2007.

[100] R. B. Patel, K. D. Prajapati, B. M. Sonara, M. Sharma, H. M. Patel, V. D. Pawar, and M. R. Jain, "Ameliorative potential of aliskiren in experimental colitis in mice," European Journal of Pharmacology, vol. 737, pp. 70-76, 2014.

[101] B. Rakesh, P. B, K. Prajapati, B. Sonara, M. Sharma, V. Pawar, and M. Jain, "Anti-cytokine and anti-hyperalgesic effects of aliskiren in experimental models of inflammation," Journal of Advanced Pharmacy Education and Research, vol. 4, p. 125, 2014.

[102] N. Noma, G. Fujii, S. Miyamoto, M. Komiya, R. Nakanishi, M. Shimura, S. Tanuma, and M. Mutoh, "Impact of acetazolamide, a carbonic anhydrase inhibitor, on the development of intestinal polyps in min mice," International Journal of Molecular Sciences, vol. 18, no. 4, p. 851, 2017.

[103] C. Jia, D. Jiang, H. Dai, F. Xiao, and C. Wang, "Pendrin, an anion exchanger on lung epithelial cells, could be a novel target for lipopolysaccharide-induced acute lung injury mice." American Journal of Translational Research, vol. 8, no. 2, pp. 981-992, 2016.

[104] F. Touret, M. Gilles, K. Barral, A. Nougairède, E. Decroly, $X$. de Lamballerie, and B. Coutard, "In vitro screening of a fda 
approved chemical library reveals potential inhibitors of sarscov-2 replication," bioRxiv, 2020.

[105] H. Blau, K. Klein, I. Shalit, D. Halperin, and I. Fabian, "Moxifloxacin but not ciprofloxacin or azithromycin selectively inhibits il-8, il-6, erk1/2, jnk, and nf-b activation in a cystic fibrosis epithelial cell line," American Journal of Physiology-lung Cellular and Molecular Physiology, vol. 292, no. 1, 2007.

[106] J. J. M. Bouwman, F. L. J. Visseren, P. K. Bouter, and R. J. A. Diepersloot, "Azithromycin inhibits interleukin-6 but not fibrinogen production in hepatocytes infected with cytomegalovirus and chlamydia pneumoniae," Journal of Laboratory and Clinical Medicine, vol. 144, no. 1, pp. 18-26, 2004.

[107] J. Liu, M. Nishida, H. Inui, J. Chang, Y. Zhu, K. Kanno, H. Matsuda, M. Sairyo, T. Okada, H. Nakaoka et al., "Rivaroxaban suppresses the progression of ischemic cardiomyopathy in a murine model of diet-induced myocardial infarction." Journal of Atherosclerosis and Thrombosis, vol. 26, no. 10, pp. 915-930, 2019.

[108] G. Monux, J. J. Zamoranoleon, P. Marques, B. Sopena, J. M. Garciagarcia, G. L. De Koller, B. Calvorico, M. A. Garciafernandez, J. A. Serrano, and A. Lopezfarre, "Fxa inhibition by rivaroxaban modifies mechanisms associated with the pathogenesis of human abdominal aortic aneurysms." British Journal of Clinical Pharmacology, vol. 83, no. 12, pp. 2661-2670, 2017.

[109] P. Kirchhof, M. D. Ezekowitz, Y. Purmah, S. Schiffer, and R. Cappato, "Effects of rivaroxaban on biomarkers of coagulation and inflammation: A post hoc analysis of the x-vert trial," TH open:companion journal to thrombosis and haemostasis, vol. 4, no. 1, pp. e20-e32, 2020.

[110] G. C. Alvarez, V. Madridmarina, R. Jimenezmendez, A. L. Buitimea, M. B. Roman, R. Cortezgomez, J. R. Esparza, and L. Rodriguezfragoso, "Combined therapy with danazol, pegilated interferon, and ribavirin improves thrombocytopenia and liver injury in rats with fibrosis," Annals of Hepatology, vol. 6, no. 4, pp. 233-241, 2007.

[111] S. G. Zarogiannis, J. W. Noah, A. Jurkuvenaite, C. Steele, S. Matalon, and D. L. Noah, "Comparison of ribavirin and oseltamivir in reducing mortality and lung injury in mice infected with mouse adapted a/california/04/2009 (h1n1)." Life Sciences, vol. 90 , no. 11, pp. 440-445, 2012.

[112] B. Morgenstern, M. Michaelis, P. C. Baer, H. W. Doerr, and J. Cinatl, "Ribavirin and interferon- synergistically inhibit sarsassociated coronavirus replication in animal and human cell lines," Biochem Biophys Res Commun, vol. 326, no. 4, pp. 905-908, 2005.

[113] W. Wang, S. Chen, I. J. Liu, C. Kao, H. Chen, B. Chiang, J. Wang, W. Sheng, P. Hsueh, C. Yang et al., "Temporal relationship of viral load, ribavirin, interleukin (il)-6, il-8, and clinical progression in patients with severe acute respiratory syndrome," Clinical Infectious Diseases, vol. 39, no. 7, pp. 1071-1075, 2004.

[114] J. G. Mastronarde, M. M. Monick, T. J. Gross, and G. W. Hunninghake, "Amiloride inhibits cytokine production in epithelium infected with respiratory syncytial virus." American Journal of Physiology-lung Cellular and Molecular Physiology, vol. 271, no. 2, p. 7, 1996.

[115] P. Fu, A. A. Birukova, J. Xing, S. Sammani, J. S. Murley, J. G. N. Garcia, D. J. Grdina, and K. G. Birukov, "Amifostine reduces lung vascular permeability via suppression of inflammatory signalling," European Respiratory Journal, vol. 33, no. 3, pp. 612-624, 2009.

[116] E. Schrezenmeier and T. Dorner, "Mechanisms of action of hydroxychloroquine and chloroquine: implications for rheumatology." Nature Reviews Rheumatology, vol. 16, no. 3, pp. 155-166, 2020.

[117] V. Lotteau, L. Teyton, A. Peleraux, T. Nilsson, L. Karlsson, S. L. Schmid, V. Quaranta, and P. A. Peterson, "Intracellular transport of class ii mhc molecules directed by invariant chain." Nature, vol. 348, no. 6302, pp. 600-605, 1990.

[118] D. Zhou, S. Dai, and Q. Tong, "Covid-19: A recommendation to examine the effect of hydroxychloroquine in preventing infection and progression," Journal of Antimicrobial Chemotherapy, 2020.

[119] J. Tang, M. Qu, M. Wang, M. Zhang, J. Yan, and Q. Mei, “Line: Large-scale information network embedding." 2015.

[120] S. Cao, W. Lu, and Q. Xu, "Grarep: Learning graph representations with global structural information," pp. 891-900, 2015

[121] L. F. R. Ribeiro, P. H. P. Saverese, and D. R. Figueiredo, "struc2vec: Learning node representations from structural identity." ACM, 2017, pp. 385-394.
[122] F. Wan, L. Hong, A. Xiao, T. Jiang, and J. Zeng, “Neodti: neural integration of neighbor information from a heterogeneous network for discovering new drugctarget interactions," Bioinformatics, vol. 35, no. 1, pp. 104-111, 2018.

[123] X. Yue, Z. Wang, J. G. Huang, S. Parthasarathy, S. Moosavinasab, Y. G. Huang, S. M. Lin, W. Zhang, P. Zhang, and H. Sun, "Graph embedding on biomedical networks: methods, applications and evaluations," Bioinformatics, vol. 36, no. 4, pp. 1241-1251, 2019.

[124] B. Perozzi, R. Alrfou, and S. Skiena, "Deepwalk: Online learning of social representations," pp. 701-710, 2014.

[125] J. Devlin, M.-W. Chang, K. Lee, and K. Toutanova, “Bert: Pretraining of deep bidirectional transformers for language understanding," arXiv preprint, p. arXiv:1810.04805, 2018.

[126] M. Ou, P. Cui, J. Pei, Z. W. Zhang, and W. W. Zhu, "Asymmetric transitivity preserving graph embedding," pp. 1105-1114, 2016.

[127] S. Kashef, H. Nezamabadipour, and B. Nikpour, "Multilabel feature selection: A comprehensive review and guiding experiments," Wiley Interdisciplinary Reviews-Data Mining and Knowledge Discovery, vol. 8, no. 2, 2018.

[128] P. Gautret, J. Lagier, P. Parola, V. T. Hoang, L. Meddeb, M. Mailhe, B. Doudier, J. Courjon, V. Giordanengo, V. E. Vieira et al., "Hydroxychloroquine and azithromycin as a treatment of covid-19: results of an open-label non-randomized clinical trial," International Journal of Antimicrobial Agents, p. 105949, 2020.

[129] X. Yao, F. Ye, M. Zhang, C. Cui, B. Huang, P. Niu, X. Liu, L. Zhao, E. Dong, C. Song et al., "In vitro antiviral activity and projection of optimized dosing design of hydroxychloroquine for the treatment of severe acute respiratory syndrome coronavirus 2 (sars-cov-2)," Clinical Infectious Diseases, 2020.

[130] J. Geleris, Y. F. Sun, J. Platt, J. Zucker, M. Baldwin, G. Hripcsak, A. Labella, D. Manson, C. Kubin, R. G. Barr, M. E. Sobieszczyk, and N. W. Schluger, "Observational study of hydroxychloroquine in hospitalized patients with covid-19," the new england journal of medicine, 2020.

[131] M. Mahevas, V. T. Tran, M. Roumier, A. Chabrol, R. Paule, C. Guillaud, S. Gallien, R. Lepeule, T. A. Szwebel, X. Lescure, F. Schlemmer, M. Matignon, M. Khellaf, E. Crickx, B. Terrier, C. Morbieu, P. Legendre, J. Dang, Y. Schoindre, J. M. Pawlotski, M. Michel, E. Perrodeau, N. Carlier, N. Roche, V. De Lastours, L. Mouthon, E. Audureau, P. Ravaud, B. Godeau, and N. Costedoat, "No evidence of clinical efficacy of hydroxychloroquine in patients hospitalized for covid-19 infection with oxygen requirement: results of a study using routinely collected data to emulate a target trial," medRxiv, 2020.

[132] G. S. Wu, J. Liu, and X. Yue, "Prediction of drug-disease associations based on ensemble meta paths and singular value decomposition," BMC bioinformatics, vol. 20, no. Suppl 3, p. 134, 2019.

[133] Y. Ge, T. Tian, S. Huang, F. Wan, J. Li, S. Li, H. Yang, L. Hong, $\mathrm{N}$. Wu, E. Yuan et al., "A data-driven drug repositioning framework discovered a potential therapeutic agent targeting covid19," bioRxiv, 2020.

[134] T. F. Smith and M. S. Waterman, "Identification of common molecular subsequences," Journal of Molecular Biology, vol. 147, no. 1, pp. 195-197, 1981.

[135] M. Hattori, Y. Okuno, S. Goto, and M. Kanehisa, "Development of a chemical structure comparison method for integrated analysis of chemical and genomic information in the metabolic pathways," Journal of the American Chemical Society, vol. 125, no. 39, pp. 11853-11865, 2003.

[136] D. Rogers and M. Hahn, "Extended-connectivity fingerprints." Journal of Chemical Information and Modeling, vol. 50, no. 5, pp. 742-754, 2010.

[137] G. Fu, Y. Ding, A. Seal, B. Chen, Y. Sun, and E. Bolton, "Predicting drug target interactions using meta-path-based semantic network analysis," BMC bioinformatics, vol. 17, p. 160, 2016.

[138] Y. Z. Sun, J. W. Han, X. F. Yan, P. Yu, and T. Y. Wu, "Pathsim: Meta path-based top-k similarity search in heterogeneous information networks," Proceedings of the VLDB Endowment, vol. 4, pp. 992 1003, 2011.

[139] A. Vaswani, N. Shazeer, N. Parmar, J. Uszkoreit, L. Jones, A. N. Gomez, L. u. Kaiser, and I. Polosukhin, "Attention is all you need," pp. 5998-6008, 2017.

[140] K. M. He, X. Y. Zhang, S. Q. Ren, and J. Sun, "Deep residual learning for image recognition," in The IEEE Conference on Computer Vision and Pattern Recognition (CVPR), 2016. 
[141] R. Reghunathan, M. Jayapal, L. Y. Hsu, H. Chng, D. Y. H. Tai, B. P. Leung, and A. J. Melendez, "Expression profile of immune response genes in patients with severe acute respiratory syndrome," BMC Immunology, vol. 6, no. 1, p. 2, 2005.

[142] M. Sirota, J. T. Dudley, J. Kim, A. P. Chiang, A. A. Morgan, A. Sweetcordero, J. Sage, and A. J. Butte, "Discovery and preclinical validation of drug indications using compendia of public gene expression data," Science Translational Medicine, vol. 3, no. 96, p. 96, 2011.

[143] L. Nanni and S. Brahnam, "Multi-label classifier based on histogram of gradients for predicting the anatomical therapeutic chemical class/classes of a given compound," Bioinformatics, vol. 33, no. 18, pp. 2837-2841, 2017.

[144] P. Goyal and E. Ferrara, "Graph embedding techniques, applications, and performance: A survey," Knowledge Based Systems, vol. 151, pp. 78-94, 2018.

[145] X. X. Zeng, S. Y. Zhu, X. R. Liu, Y. D. Zhou, R. Nussinov, and F. X. Cheng, "deepdr: a network-based deep learning approach to in silico drug repositioning," Bioinformatics, vol. 35, no. 24, pp. 5191-5198, 2019.

[146] Y. Luo, X. B. Zhao, J. T. Zhou, J. L. Yang, Y. Q. Zhang, W. H. Kuang, J. Peng, L. G. Chen, and J. Y. Zeng, "A network integration approach for drug-target interaction prediction and computational drug repositioning from heterogeneous information," Nature communications, vol. 8, no. 1, p. 573, 2017.

[147] N. Natarajan and I. S. Dhillon, "Inductive matrix completion for predicting genecdisease associations," Bioinformatics, vol. 30, no. 12 , pp. i60-i $68,2014$. 